\title{
Asymptotic behaviour for a class of non-monotone delay differential systems with applications
}

\author{
Teresa Faria回句, Rafael Obayad, Ana M. Sanz d
}

Suggested running head: Asymptotic behaviour for a class of non-monotone DDEs

\begin{abstract}
The paper concerns a class of $n$-dimensional non-autonomous delay differential equations obtained by adding a non-monotone delayed perturbation to a linear homogeneous cooperative system of ordinary differential equations. This family covers a wide set of models used in structured population dynamics. By exploiting the stability and the monotone character of the linear ODE, we establish sufficient conditions for both the extinction of all the populations and the permanence of the system. In the case of DDEs with autonomous coefficients (but possible time-varying delays), sharp results are obtained, even in the case of a reducible community matrix. As a subproduct, our results improve some criteria for autonomous systems published in recent literature. As an important illustration, the extinction, persistence and permanence of a non-autonomous Nicholson system with patch structure and multiple time-dependent delays are analysed.
\end{abstract}

Keywords: delay differential equation; non-autonomous Nicholson system; quasi-monotone condition; persistence; permanence; global asymptotic stability.

2010 Mathematics Subject Classification: 34K25, 34K12, 34K27, 34K20, 92D25.

\section{Introduction}

In the last decades, delay differential equations (DDEs) with patch structure have been largely employed in population dynamics and other fields, since by capturing several features of a heterogeneous environment, they may provide quite realistic models. Structured systems of differential equations have been used in population models when the populations are distributed over different classes (e.g. due to age, size or different food-rich patches), in disease models with several compartments

\footnotetext{
${ }^{\mathrm{a}}$ Corresponding author.

b Departamento de Matemática and CMAF-CIO, Faculdade de Ciências, Universidade de Lisboa, Campo Grande, 1749-016 Lisboa, Portugal teresa.faria@fc.ul.pt

c Departamento de Matemática Aplicada, E. Ingenierías Industriales, and IMUVA, Instituto de Matemáticas, Universidad de Valladolid, 47011 Valladolid, Spain rafoba@wmatem.eis.uva.es

d Departamento de Didáctica de las Ciencias Experimentales, Sociales y de la Matemática, Facultad de Educación, and IMUVA, Instituto de Matemáticas, Universidad de Valladolid, 34004 Palencia, Spain anasan@wmatem.eis.uva.es
} 
for a host population, in leukemia models where the infected cells may become inactive, and in a variety of other situations where the transition among the several classes should be considered. See e.g. [6, 20, 25, 26]. Naturally, time delays should be incorporated in such systems to express the maturation time of biological species, the incubation period of diseases, the maturation time of blood cells and several other attributes.

The paper is concerned with a family of non-autonomous DDEs written in abstract form as

$$
x^{\prime}(t)=A(t) x(t)+f\left(t, x_{t}\right), \quad t \geq 0
$$

where $A(t)$ is an $n \times n$ matrix of continuous functions, $f:[0, \infty) \times D \rightarrow \mathbb{R}^{n}$ is continuous, $D \subset$ $C\left([-\tau, 0] ; \mathbb{R}^{n}\right)$ is equipped with the uniform convergence metric, $\tau>0$ is the time-delay, and, as usual, $x_{t}$ denotes the past history of the system on the interval $[t-\tau, t]$, i.e., $x_{t}(\theta)=x(t+\theta)$ for $\theta \in[-\tau, 0]$. The function $f$ is required to satisfy $f(t, 0)=0$ for $t \geq 0$, and the ordinary differential equation (ODE) $x^{\prime}(t)=A(t) x(t)$ to be cooperative, thus $a_{i j}(t) \geq 0$ must hold for all $i \neq j$ and $t \geq 0$, where $a_{i j}(t)$ are the entries of $A(t)$. We restrict our setting to a class of delayed perturbations $f\left(t, x_{t}\right)$ with multiple time-varying discrete delays, having the particular form

$$
f(t, \phi)=\left(f_{1}\left(t, \phi_{1}\right), \ldots, f_{n}\left(t, \phi_{n}\right)\right)
$$

for $t \geq 0$ and $\phi=\left(\phi_{1}, \ldots, \phi_{n}\right) \in D$, where $f_{i}\left(t, \phi_{i}\right)=\sum_{k=1}^{m} n_{i k}\left(t, \phi_{i}\left(-\tau_{i k}(t)\right)\right)$ and $n_{i k}(t, x), \tau_{i k}(t)$ are continuous, bounded and nonnegative functions, for all $i, k$. For simplicity, this paper deals with discrete delays only; however, as pointed out later in Section 3, straightforward generalizations to some families of perturbations with distributed delays are possible.

Inserting (1.2) in (1.1) leads to systems of the form

$$
x_{i}^{\prime}(t)=\sum_{j=1}^{n} a_{i j}(t) x_{j}(t)+\sum_{k=1}^{m} n_{i k}\left(t, x_{i}\left(t-\tau_{i k}(t)\right)\right), i=1, \ldots, n, t \geq 0,
$$

which can be interpreted as a structured population model for $n$ populations, see Section 3 for an additional set of hypotheses, as well as for some biological elements of the model.

In the present paper, the main idea is to take full advantage of the properties of the cooperative non-delayed linear system $x^{\prime}(t)=A(t) x(t)$, to further analyse the large-time behaviour of solutions of system (1.3). We shall impose conditions on the coefficients of the linear system $x^{\prime}(t)=A(t) x(t)$, in order to have its global exponential stability. This property and the monotonicity of $x^{\prime}(t)=A(t) x(t)$ will play an important role in the study of (1.3). Although the nonlinearities (1.2) are in general nonmonotone, the techniques exploited here are largely based on results of comparison of solutions (see [24]), applied to some convenient auxiliary cooperative DDE systems. This method is used to address the global asymptotic behaviour of solutions of system (1.3), in what concerns its dissipativity, uniform persistence and the global asymptotic stability of the null solution. To some extent and in different frameworks, similar techniques have inspired the papers [8, 9, 16, 17, 28. Some relevant applications are given. We also hope that the present results can be used to further address other aspects of the global dynamics of (1.3).

As a significant example of systems in the form (1.3), we shall consider a non-autonomous Nicholson system with patch structure and multiple time-dependent discrete delays, given by

$$
x_{i}^{\prime}(t)=-d_{i}(t) x_{i}(t)+\sum_{j=1, j \neq i}^{n} a_{i j}(t) x_{j}(t)+\sum_{k=1}^{m} \beta_{i k}(t) x_{i}\left(t-\tau_{i k}(t)\right) e^{-c_{i k}(t) x_{i}\left(t-\tau_{i k}(t)\right)}, i=1, \ldots, n,
$$


where all the coefficient and delay functions are continuous, nonnegative and bounded. We stress that results concerning multi-dimensional versions of the famous Nicholson's blowflies equation [13] are still quite limited, with most authors treating only autonomous systems.

The papers of Faria and Röst [10], on autonomous Nicholson systems, and of Obaya and Sanz [22], on uniform and strict persistence for monotone skew-product semiflows, were a strong motivation for the present work. Here, the authors further pursue their previous research, and extend it to general non-autonomous Nicholson systems: in fact, we aim to obtain results on extinction, uniform persistence and permanence of (1.4) as simple illustrations of our main results, proven for a much larger family of DDEs of the form (1.3).

The contents of the paper are now briefly described. In Section 2, we study a cooperative ODE $x^{\prime}(t)=A(t) x(t)$ and give sufficient conditions for its global exponential stability; in this case, a system of the form (1.1) is dissipative if the delayed perturbation $f\left(t, x_{t}\right)$ is bounded. In Section 3, we start by introducing a set of assumptions for a family of DDEs (1.1), give some biological interpretation of the models and refer to some recent literature. The main results are then presented, providing very general criteria for both the global asymptotic stability of the trivial solution (in biological terms, this implies the extinction of the populations in all patches) and the uniform persistence of such systems. A comparison with results in [9, 18, 28, 30] is also given, and some questions are raised to be left as open problems. Finally, in Section 4 we consider systems with autonomous coefficients (but with possible time-dependent delays): from the results in Section 3 and by a careful analysis of properties of cooperative matrices, we provide necessary and sufficient conditions for both their permanence and extinction, even in the case of a reducible community matrix. These sharp criteria improve and extend results for autonomous systems proven in recent literature. As an important example of application, throughout the paper our results are widely illustrated with versions of the Nicholson system (1.4).

\section{Preliminaries}

In this section, we establish some preliminary results on stability for non-autonomous linear homogeneous systems of ODEs of cooperative type. Although such systems have been widely studied (see e.g. [5, 12, 14]), some optimal conditions for their asymptotic stability and global exponential stability are given here. For completeness of the reader, the authors opt to include these conditions here, with the proof of a result whenever its precise statement could not be found elsewhere. We start with some standard definitions from the literature [5, 12, 14].

Consider an $n$-dimensional ODE $x^{\prime}=f(t, x)$ with $f:[\alpha, \infty) \times D \subset \mathbb{R}^{n+1} \rightarrow \mathbb{R}^{n}$ sufficiently regular so that for any $\left(t_{0}, x_{0}\right) \in[\alpha, \infty) \times D$ there exists a unique solution, denoted by $x\left(t, t_{0}, x_{0}\right)$, of the initial value problem $x^{\prime}=f(t, x), x\left(t_{0}\right)=x_{0}$, defined on $[\alpha, \infty)$. To simplify the writing, let $D=\mathbb{R}^{n}$. We further assume that $x=0$ is a solution, i.e., $f(t, 0)=0, t \geq \alpha$. The zero solution is said to be stable on the interval $[\alpha, \infty)$ if for any $\varepsilon>0$ and $t_{0} \geq \alpha$ there is $\delta=\delta\left(\varepsilon, t_{0}\right)>0$ such that $\left|x\left(t, t_{0}, x_{0}\right)\right|<\varepsilon$ for all $t \geq t_{0}$, whenever $\left|x_{0}\right|<\delta ; x=0$ is uniformly stable if it is stable and $\delta$ above can be chosen independently of $t_{0} \geq \alpha$. The zero solution is said to be uniformly asymptotically stable on $[\alpha, \infty)$ if it is uniformly stable and there is $b>0$ such that, for any $\varepsilon>0$, there is $T=T(\varepsilon)>\alpha$ such that, for any $t_{0} \geq \alpha$ and $\left|x_{0}\right|<b$, we have $\left|x\left(t, t_{0}, x_{0}\right)\right|<\varepsilon$ for all $t \geq t_{0}+T$; and $x=0$ is globally exponentially stable on $[\alpha, \infty)$ if there exist $K, \beta>0$ such that $\left|x\left(t, t_{0}, x_{0}\right)\right| \leq K e^{-\beta\left(t-t_{0}\right)}\left|x_{0}\right|$ 
for all $t \geq t_{0} \geq \alpha$ and $x_{0} \in \mathbb{R}^{n}$. In general, the stability of a particular solution $\tilde{x}(t)$ of an ODE $x^{\prime}=f(t, x)$ is defined as the stability of the zero solution of $y^{\prime}=f(t, \tilde{x}+y)-f(t, \tilde{x})$.

The usual partial order in $\mathbb{R}^{n}$ relative to the cone $[0, \infty)^{n}$ is denoted here by $\leq$, i.e., for $x, y \in \mathbb{R}^{n}$, $x \leq y$ means $y-x \in[0, \infty)^{n}$; we write $x \ll y$ whenever $y-x \in(0, \infty)^{n}$. The notations $\geq$ and $\gg$ have then a clear meaning. In particular, a vector $v$ in $\mathbb{R}^{n}$ is said to be positive (nonnegative) if all its components are positive (nonnegative), and we write $v \gg 0(v \geq 0)$; by $v>0$ we mean that $v \geq 0$ and $v \neq 0$.

Lemma 2.1. Consider a non-autonomous linear $O D E$

$$
x^{\prime}(t)=A(t) x(t), \quad t \geq \alpha,
$$

where $\alpha \in \mathbb{R}$ and $A(t)=\left[a_{i j}(t)\right]$ is an $n \times n$ matrix of functions such that:

(a1) $a_{i j}$ are continuous on $[\alpha, \infty), a_{i j}(t) \geq 0, i \neq j, a_{i i}(t)<0$ for all $t \geq \alpha$ and $i, j \in\{1, \ldots, n\}$;

(a2) there exists a vector $v=\left(v_{1}, \ldots, v_{n}\right) \gg 0$ such that $A(t) v \leq 0$ for all $t \geq \alpha$.

Then, for any solution $x(t)$ of (2.1), $|x(t)|_{v^{-1}}$ is non-increasing on $t \in[\alpha, \infty)$, where $|\cdot|_{v^{-1}}$ is the norm in $\mathbb{R}^{n}$ defined by $|x|_{v^{-1}}=\max _{1 \leq i \leq n}\left(v_{i}^{-1}\left|x_{i}\right|\right)$ for $x=\left(x_{1}, \ldots, x_{n}\right)$.

Proof. Rescaling the variables by $\hat{x}_{i}(t)=v_{i}^{-1} x_{i}(t)(1 \leq i \leq n)$, where $v=\left(v_{1}, \ldots, v_{n}\right) \gg 0$ is a vector as in (a2), we obtain a new linear $\operatorname{ODE} \hat{x}^{\prime}(t)=\hat{A}(t) \hat{x}(t)$, where the matrix $\hat{A}(t)=\left[\hat{a}_{i j}(t)\right]$ has entries $\hat{a}_{i j}(t)=v_{i}^{-1} a_{i j}(t) v_{j}$. In this way, and after dropping the hats for simplicity, we may consider (2.1) where $v=\mathbf{1}:=(1, \ldots, 1)$ is the positive vector in (a2) and $|x|_{v^{-1}}=\max _{1 \leq i \leq n}\left|x_{i}\right|$.

Let $x(t) \neq 0$ be a solution of (2.1). To prove the claim, we show that $|x(t)|$ is non-increasing on each fixed interval $J=\left[t_{0}, t_{1}\right], \alpha \leq t_{0}<t_{1}$. Define $u_{j}=\max _{J}\left|x_{j}(t)\right|$, and let $u_{i}=\max _{1 \leq j \leq n} u_{j}$, with $u_{i}=\left|x_{i}\left(t_{*}\right)\right|$ for some $t_{*} \in J$. It is sufficient to show that $u_{i}=\left|x_{i}\left(t_{0}\right)\right|$.

We suppose that $x_{i}\left(t_{*}\right)>0$; the case $x_{i}\left(t_{*}\right)<0$ is treated in a similar way. Denoting $d_{i}(t)=$ $-a_{i i}(t)$ and $D_{i}(t)=\int_{t_{0}}^{t} d_{i}(s) d s$, for $t \in J$ we have $x_{i}^{\prime}(t)+d_{i}(t) x_{i}(t) \leq d_{i}(t) u_{i}$. Hence

$$
x_{i}(t) \leq x_{i}\left(t_{0}\right) e^{-D_{i}(t)}+u_{i}\left(1-e^{-D_{i}(t)}\right), \quad t \in J .
$$

In particular for $t=t_{*}$ we derive $u_{i} e^{-D_{i}\left(t_{*}\right)} \leq x_{i}\left(t_{0}\right) e^{-D_{i}\left(t_{*}\right)}$, thus $u_{i}=x_{i}\left(t_{0}\right)$.

Lemma 2.2. For the linear ODE system (2.1), assume

(a1') $a_{i j}$ are uniformly continuous and bounded on $[\alpha, \infty), a_{i j}(t) \geq 0, i \neq j, a_{i i}(t)<0$ for all $t \geq \alpha$ and $i, j \in\{1, \ldots, n\}$;

(a2') there exists a vector $v=\left(v_{1}, \ldots, v_{n}\right) \gg 0$ such that $A(t) v \leq 0$ for all $t \geq \alpha$, and $\liminf _{t \rightarrow \infty} A(t) v \ll 0$, in the sense that there exists a sequence $t_{k} \rightarrow \infty$ such that $\lim _{k}\left(A\left(t_{k}\right) v\right)_{i}<$ $0, i=1, \ldots, n$.

Then, (2.1) is asymptotically stable; in other words, $x=0$ is stable and $\lim _{t \rightarrow \infty} x(t)=0$, for all solutions of (2.1).

Proof. As in the above proof and without loss of generality, consider $v=\mathbf{1}$ in (a2') and the norm $|x|=\max _{1 \leq i \leq n}\left|x_{i}\right|$ in $\mathbb{R}^{n}$. From Lemma 2.1, (2.1) is uniformly stable. We now prove that the trivial solution is a global attractor of all solutions.

Let $x(t) \neq 0$ be a solution of (2.1), and define $c=\lim _{t \rightarrow \infty}|x(t)|$. We want to show that $c=0$. In order to obtain a contradiction, suppose that $c>0$. By (a2'), take $t_{k} \rightarrow \infty$ such that 
$\alpha_{i}:=\lim _{k}\left(-d_{i}\left(t_{k}\right)+\sum_{j \neq i} a_{i j}\left(t_{k}\right)\right)<0$ for all $i$. In particular for such a sequence, $\left|x\left(t_{k}\right)\right| \searrow c$, and thus there exists $i \in\{1, \ldots, n\}$ and a subsequence, still denoted by $\left(t_{k}\right)$, such that either $x_{i}\left(t_{k}\right)=\left|x\left(t_{k}\right)\right| \rightarrow c$ or $x_{i}\left(t_{k}\right)=-\left|x\left(t_{k}\right)\right| \rightarrow-c$. We only consider the situation $x_{i}\left(t_{k}\right) \rightarrow c$ for some $i$, the other is treated in a similar way. We now consider separately two cases.

First, suppose that there exists $\lim _{t \rightarrow \infty} x_{i}(t)=c$. Since the entries of $A(t)$ are bounded and uniformly continuous and $x(t)$ is uniformly bounded on $[\alpha, \infty)$, one easily shows that all components $x_{j}(t)$ and $x_{j}^{\prime}(t)$ are uniformly continuous on $[\alpha, \infty)$. From the Barbalat Lemma, we derive that there is $\lim _{t \rightarrow \infty} x_{i}^{\prime}(t)=0$, and in particular obtain $\lim _{k} x_{i}^{\prime}\left(t_{k}\right)=0$. On the other hand, from (2.1) we have

$$
x_{i}^{\prime}\left(t_{k}\right) \leq x_{i}\left(t_{k}\right)\left[-d_{i}\left(t_{k}\right)+\sum_{j \neq i} a_{i j}\left(t_{k}\right)\right] .
$$

Taking limits, the above inequality leads to $0 \leq c \alpha_{i}$, which is a contradiction.

Next, consider the case when $\underline{x}_{i}:=\liminf _{t \rightarrow \infty} x_{i}(t)<\limsup _{t \rightarrow \infty} x_{i}(t)=c$. From the inequality above, it is clear that $t_{k}$ are not local extrema points, since $x_{i}^{\prime}\left(t_{k}\right)<0$. However, by reducing to a subsequence if necessary, we may consider that each $t_{k}$ lies between a local maximum point $\bar{t}_{k}$ and a local minimum point $\underline{t}_{k}$, to its left and to its right respectively, with $x_{i}\left(\bar{t}_{k}\right) \rightarrow c, x_{i}\left(\underline{t}_{k}\right) \rightarrow \underline{x}_{i}$. We may take a sequence $\left(s_{k}\right)$ such that, for all $k \in \mathbb{N}$,

$$
t_{k}<\underline{t}_{k}<s_{k}<\bar{t}_{k+1}<t_{k+1}, \quad x_{i}\left(s_{k}\right)=x_{i}\left(t_{k}\right), \quad x_{i}^{\prime}\left(s_{k}\right) \geq 0 .
$$

Since the map $t \rightarrow|x(t)|$ is non-increasing, we have $x_{j}\left(s_{k}\right) \leq\left|x\left(s_{k}\right)\right| \leq\left|x\left(t_{k}\right)\right|=x_{i}\left(t_{k}\right), 1 \leq j \leq n$. We derive

$$
0 \leq x_{i}^{\prime}\left(s_{k}\right) \leq x_{i}\left(t_{k}\right)\left[-d_{i}\left(s_{k}\right)+\sum_{j \neq i} a_{i j}\left(s_{k}\right)\right] \leq 0,
$$

and therefore $x_{i}\left(t_{k}\right)=0$, which is not possible.

When $f(t, x)$ is periodic in $t$, a solution of $x^{\prime}=f(t, x)$ is uniformly asymptotically stable if it is asymptotically stable. This is not true if periodic is replaced by almost periodic (see [12], p. 191 for a counter-example). Moreover, for a linear system $x^{\prime}=A(t) x$, where $A(t)$ is an $n \times n$ matrix of continuous functions, it is well known that the concepts of global exponential stability and uniform asymptotic stability on an interval $[\alpha, \infty)$ are equivalent (see [5, 14]). Therefore, the following criterion is straightforward for periodic systems, however it applies to the more general case of almost periodic linear systems.

Theorem 2.1. Let $A(t)=\left[a_{i j}(t)\right]$ be an $n \times n$ matrix of almost periodic functions on $\mathbb{R}$ satisfying (a1), (a2) on $\mathbb{R}$, with $A\left(t_{0}\right) v \ll 0$ for some $t_{0} \in \mathbb{R}$. Then, (2.1) is globally exponentially stable.

Proof. Let $H(A)$ be the hull of $A$, that is, the closure for the topology of uniform convergence of the set of shifted maps $\left\{\theta_{t} A(\cdot)=A(\cdot+t) \mid t \in \mathbb{R}\right\}$ [5, 12]. $H(A)$ is a compact metric space. Since $A$ is almost periodic, it follows that $A$ satisfies (a1'), (a2'). The orbit $\left\{\theta_{t} A \mid t \in \mathbb{R}\right\}$ is dense in the hull, thus actually any $B \in H(A)$ satisfies (a1'), (a2') as well. By Lemma 2.2, all solutions of all the systems $x^{\prime}=B(t) x$, with $B \in H(A)$, tend to 0 as $t \rightarrow \infty$. At this point, the spectral theory of Sacker and Sell [23] applies and permits to conclude that (2.1) is globally exponentially stable. 
Usually, the global exponential stability of (2.1) is obtained by assuming that $A(t)$ is strongly uniformly row (or column) dominant. The theorem below follows from Proposition 6.3 in [5].

Theorem 2.2. Consider an $n \times n$ matrix $A(t)=\left[a_{i j}(t)\right]$ of bounded continuous functions satisfying (a1), and suppose that

(a3) there exist a vector $v=\left(v_{1}, \ldots, v_{n}\right) \gg 0$ and $T \geq \alpha, \delta>0$ such that $(A(t) v)_{i} \leq-\delta$ for all $t \geq T, i=1, \ldots, n$.

Then, (2.1) is globally exponentially stable.

Remark 2.1. For any fixed $t$, the matrix $-A(t)$ is a non-singular M-matrix if and only if there exists a positive vector $v=v(t)$ such that $A(t) v \ll 0$; thus, condition (a3) above not only demands that $-A(t)$ are non-singular M-matrices, for $t$ sufficiently large, but also that there exist positive vectors $v, \eta$, which do not depend on $t$, such that $A(t) v \leq-\eta$ (see [11] and Section 4 for more details on M-matrices).

For $\tau \geq 0$, consider the Banach space $C:=C\left([-\tau, 0] ; \mathbb{R}^{n}\right)$ equipped with the norm $\|\phi\|=$ $\max _{\theta \in[-\tau, 0]}|\phi(\theta)|$, where $|\cdot|$ is a fixed norm in $\mathbb{R}^{n}$. The case of no delays $(\tau=0)$ is included, in which case $C$ is identified with $\mathbb{R}^{n}$. We now consider DDEs obtained by adding a bounded delayed perturbation $f\left(t, x_{t}\right)$ to systems (2.1), where, as before, $x_{t} \in C$ is given by $x_{t}(\theta)=x(t+\theta),-\tau \leq$ $\theta \leq 0$. For simplicity, in what follows we take $\alpha=0$, but any $\alpha \in \mathbb{R}$ could be considered.

From Theorem 2.2, one obtains:

Theorem 2.3. Consider an $n \times n$ matrix $A(t)=\left[a_{i j}(t)\right]$ of bounded functions satisfying (a1), (a3) on $[0, \infty)$, and a function $f:[0, \infty) \times C \rightarrow \mathbb{R}^{n}$ continuous and bounded. Then, all solutions of the $D D E$

$$
x^{\prime}(t)=A(t) x(t)+f\left(t, x_{t}\right), \quad t \geq 0,
$$

are defined on $[0, \infty)$ and (2.2) is dissipative, i.e., there exists $M>0$ such that $\limsup _{t \rightarrow \infty}|x(t)| \leq M$ for any solution $x(t)$ of (2.2).

Proof. Let $|f(t, \varphi)| \leq L$ for $t \geq 0, \varphi \in C$. From Theorem 2.2. there are $K>0, \alpha>0$ such that $\left|X(t) X^{-1}\left(t_{0}\right)\right| \leq K e^{-\alpha\left(t-t_{0}\right)}, t \geq t_{0} \geq 0$, where $X(t)$ is a fundamental solution matrix for (2.1). By the variation of constants formula, the solutions $x(t)$ of (2.2) satisfy

$$
x(t)=X(t) X^{-1}\left(t_{0}\right) x\left(t_{0}\right)+X(t)\left(\int_{t_{0}}^{t} X^{-1}(s) f\left(s, x_{s}\right) d s\right) \quad\left(t, t_{0} \geq 0\right),
$$

so that $|x(t)| \leq K e^{-\alpha\left(t-t_{0}\right)}\left|x\left(t_{0}\right)\right|+\frac{K L}{\alpha}\left(1-e^{-\alpha\left(t-t_{0}\right)}\right) \rightarrow \frac{K L}{\alpha} \quad$ as $\quad t \rightarrow \infty$.

We now set some further notation. Let $C^{+}$be the cone of nonnegative functions in $C, C^{+}=$ $C\left([-\tau, 0] ;[0, \infty)^{n}\right)$, and $\operatorname{int} C^{+}$its interior. Hereafter, $\leq$also denotes the usual partial order generated by $C^{+}: \phi \leq \psi$ if and only if $\psi-\phi \in C^{+}$; by $\phi \ll \psi$, we mean that $\psi-\phi \in$ int $C^{+}$. The definition of the relations $\geq$ and $\gg$ are then clear; thus, we write $\psi \geq 0$ for $\psi \in C^{+}$and $\psi \gg 0$ for $\psi \in \operatorname{int} C^{+}$. A vector $v \in \mathbb{R}^{n}$ is identified in $C$ with the constant function $\psi(s)=v$ for $-\tau \leq s \leq 0$.

Let $D \subset C\left([-\tau, 0] ; \mathbb{R}^{n}\right)(\tau \geq 0)$ be open, and consider a non-autonomous DDE written as

$$
x^{\prime}(t)=f\left(t, x_{t}\right), \quad t \geq 0,
$$


where $f:[0, \infty) \times D \rightarrow \mathbb{R}^{n}$ is continuous and regular enough so that the initial value problem is well-posed, in the sense that for each $(\sigma, \phi) \in[0, \infty) \times D$ there exists a unique solution of the problem $x^{\prime}(t)=f\left(t, x_{t}\right), x_{\sigma}=\phi$, defined on a maximal interval of existence. This solution will be denoted by $x(t, \sigma, \phi)$ in $\mathbb{R}^{n}$ or $x_{t}(\sigma, \phi)$ in $C$. When considering more than one DDE $x^{\prime}(t)=f\left(t, x_{t}\right)$, the notation $x(t, \sigma, \phi, f)$ where the argument $f$ is made explicit will be used to clarify which DDE is being considered.

To simplify the terminology, we say that (2.4) is cooperative if it satisfies Smith's quasi-monotone condition (Q), given by (see [24])

(Q) for $\phi, \psi \in D, \phi \leq \psi$ and $\phi_{i}(0)=\psi_{i}(0)$, then $f_{i}(t, \phi) \leq f_{i}(t, \psi), i=1, \ldots, n, t \geq 0$.

It is well-known that $(\mathrm{Q})$ guarantees monotonocity of solutions relative to initial data and allows comparison of solutions between two related DDEs, $x^{\prime}(t)=f\left(t, x_{t}\right), x^{\prime}(t)=g\left(t, x_{t}\right)$ with $f \leq g$ : if at least one of them is cooperative, then $x(t, \sigma, \phi, f) \leq x(t, \sigma, \psi, g)$ for $t \geq \sigma$ if $\phi \leq \psi([24])$. These and other properties of cooperative ODEs and DDEs will turn out to be very useful in the next sections. The lemma below will be often applied, see p. 82 of [24].

Lemma 2.3. 24] Consider (2.4) in $D \subset C\left([-\tau, 0] ; \mathbb{R}^{n}\right)$, and let $v=\left(v_{1}, \ldots, v_{n}\right) \in \mathbb{R}^{n}$.

(i) If $f_{i}(t, \phi) \leq 0$ for all $i=1, \ldots, n, t \geq 0$ whenever $\phi \in D, \phi \leq v$ and $\phi_{i}(0)=v_{i}$, then the set $\{\phi \in D: \phi \leq v\}$ is positively invariant for (2.4).

(ii) If $f_{i}(t, \phi) \geq 0$ for all $i=1, \ldots, n, t \geq 0$ whenever $\phi \in D, \phi \geq v$ and $\phi_{i}(0)=v_{i}$, then the set $\{\phi \in D: \phi \geq v\}$ is positively invariant for (2.4).

Remark 2.2. Clearly, if (a1) is satisfied, then (2.1) is a cooperative system and the nonnegative cone $[0, \infty)^{n}$ is forward invariant. If in addition (a2) is satisfied and $v=\left(v_{1}, \ldots, v_{n}\right) \gg 0$ is as in (a2), for $x \in \mathbb{R}^{n}$ such that $x \leq v$ and $x_{i}=v_{i}$, then $(A(t) x)_{i} \leq 0$. This implies that the interval $[0, v]:=\left[0, v_{1}\right] \times \cdots \times\left[0, v_{n}\right]$ is forward invariant as well.

\section{Global behaviour for a class of non-monotone and non-autonomous DDEs}

In this section, we consider $n$-dimensional delayed structured models (1.1), where the linear ODE system (2.1) is globally exponentially stable, $f$ is continuous, bounded, and, in general, non-monotone. Although some generalizations are possible, we restrict our framework to perturbations $f\left(t, x_{t}\right)=$ $\left(f_{1}\left(t, x_{1, t}\right), \ldots, f_{n}\left(t, x_{n, t}\right)\right)$, with each component $f_{i}\left(t, \phi_{i}\right)$ of the form $f_{i}\left(t, \phi_{i}\right)=\sum_{k=1}^{m} n_{i k}\left(t, \phi_{i}\left(-\tau_{i k}(t)\right)\right.$, for $t \geq 0, \phi=\left(\phi_{1}, \ldots, \phi_{n}\right) \in C$. Moreover, we suppose that $n_{i k}(t, 0)=0$ for $t \geq 0$ and have partial derivative with respect to the second variable at $x=0^{+}$given by $\frac{\partial n_{i k}}{\partial x}(t, 0)=\beta_{i k}(t) \geq 0$; thus $n_{i k}(t, x)$ is written as $n_{i k}(t, x)=\beta_{i k}(t) h_{i k}(t, x)$ with $h_{i k}(t, 0)=0, \frac{\partial h_{i k}}{\partial x}(t, 0)=1, t \geq 0$. Below, some additional assumptions on $n_{i k}(t, x)$ will be imposed. This leads to a non-autonomous system with multiple discrete time-dependent delays of the form

$$
x_{i}^{\prime}(t)=-d_{i}(t) x_{i}(t)+\sum_{j=1, j \neq i}^{n} a_{i j}(t) x_{j}(t)+\sum_{k=1}^{m} \beta_{i k}(t) h_{i k}\left(t, x_{i}\left(t-\tau_{i k}(t)\right)\right), i=1, \ldots, n, t \geq 0 .
$$

Throughout the remainder of this paper, either the whole or a part of the following set of hypotheses will be imposed: 
(h1) the functions $d_{i}, a_{i j}(j \neq i)$ are continuous and bounded, with $a_{i j}(t) \geq 0, i \neq j, d_{i}(t)>0$ for $t \geq 0$ and $i, j \in\{1, \ldots, n\}$

(h2) there exist a vector $v=\left(v_{1}, \ldots, v_{n}\right) \gg 0$ and $\delta>0, T_{0} \geq 0$ such that $d_{i}(t) v_{i} \geq \sum_{j=1, j \neq i}^{n} a_{i j}(t) v_{j}+$ $\delta$ for $t \geq T_{0}, i \in\{1, \ldots, n\}$;

(h3) $\tau_{i k}, \beta_{i k}$ are continuous and bounded, with $\tau_{i k}(t) \geq 0, \beta_{i k}(t) \geq 0$ and

$$
\beta_{i}(t):=\sum_{k=1}^{m} \beta_{i k}(t)>0
$$

for $t \in[0, \infty), i \in\{1, \ldots, n\}, k \in\{1, \ldots, m\}$;

(h4) $h_{i k}:[0, \infty) \times[0, \infty) \rightarrow[0, \infty)$ are bounded, continuous, $h_{i k}(t, x)$ are locally Lipschitzian in $x$, with

$$
h_{i}^{-}(x) \leq h_{i k}(t, x) \leq h_{i}^{+}(x), \quad t, x \geq 0, k=1, \ldots, m,
$$

where $h_{i}^{ \pm}:[0, \infty) \rightarrow[0, \infty)$ are continuous on $[0, \infty)$ and continuously differentiable in a vicinity of $0^{+}$, with $h_{i}^{ \pm}(0)=0,\left(h_{i}^{ \pm}\right)^{\prime}(0)=1$ and $h_{i}^{-}(x)>0$ for $x>0, i \in\{1, \ldots, n\}$.

For simplicity, here we only treat non-autonomous systems with discrete non-autonomous delays, but our framework applies with straightforward adjustments to the more general case of systems with multiple distributed time-varying delays of the form

$$
x_{i}^{\prime}(t)=-d_{i}(t) x_{i}(t)+\sum_{j=1, j \neq i}^{n} a_{i j}(t) x_{j}(t)+f_{i}\left(t, x_{i, t}\right), i=1, \ldots, n,
$$

with $f_{i}\left(t, x_{i, t}\right)$ given by

$$
f_{i}\left(t, x_{i, t}\right)=\sum_{k=1}^{m} \beta_{i k}(t) h_{i k}\left(t, L_{i k}\left(t, x_{i, t}\right)\right) \text { or } f_{i}\left(t, x_{i, t}\right)=\sum_{k=1}^{m} \beta_{i k}(t) L_{i k}\left(t, h_{i k}\left(\cdot, x_{i, t}(\cdot)\right)\right),
$$

where

$$
L_{i k}(t, \phi)=\int_{-\tau}^{0} \phi(s) d_{s} \eta_{i k}(t, s) \quad \text { for } t \geq 0, \phi \in C([-\tau, 0], \mathbb{R}),
$$

$\tau>0$, the measurable functions $\eta_{i k}:[0, \infty) \times[-\tau, 0] \rightarrow \mathbb{R}$ are continuous on $t$, with $\eta_{i k}(t, \cdot)$ nondecreasing and normalized so that $\int_{-\tau}^{0} d_{s} \eta_{i k}(t, s)=1, i=1, \ldots, n, k=1, \ldots, m, t \geq 0$, and for which (h3), (h4) hold. Besides (3.3), and under some natural conditions, other forms of dependence on distributed delays can be incorporated in (3.2).

In what follows, we refer to the $n \times n$ matrix-valued functions defined on $[0, \infty)$ by

$$
\begin{array}{ll}
D(t)=\operatorname{diag}\left(d_{1}(t), \ldots, d_{n}(t)\right), & A(t)=\left[a_{i j}(t)\right] \\
B(t)=\operatorname{diag}\left(\beta_{1}(t), \ldots, \beta_{n}(t)\right), & M(t)=B(t)+A(t)-D(t), \quad t \geq 0,
\end{array}
$$

where $a_{i i}(t) \equiv 0$. The matrix $M(t)$ is often designated as the community matrix of the population system (3.1). 
Remark 3.1. We stress that under (h1), (h2) the linear homogeneous ODE $x^{\prime}(t)=-[D(t)-$ $A(t)] x(t)$ possesses two important features: it is cooperative and globally exponentially stable. Of course, if $A(t)$ is periodic or almost periodic, Theorem 2.1 allows us to replace (h2) by the weaker condition $[D(t)-A(t)] v \geq 0$ for $t \in \mathbb{R}$ and $\left[D\left(t_{0}\right)-A\left(t_{0}\right)\right] v \gg 0$ for some $t_{0} \in \mathbb{R}$ and $v \gg 0$.

System (3.1) can be interpreted as a model for $n$ populations structured into $n$ classes or patches, with migration among them: $x_{i}(t)$ denotes the density of the $i$ th population; $a_{i j}(t)$ is the migration rate of the population in class $j$ moving to class $i ; d_{i}(t)$ is the coefficient of instantaneous loss for class $i$, which incorporates both the death rate and the emigration rates of the population that leaves class $i$ to move to other classes; the birth contribution for each population is given by the nonlinear terms $\sum_{k} \beta_{i k}(t) h_{i k}\left(t, x_{i}\left(t-\tau_{i k}(t)\right)\right)$.

With this interpretation, $d_{i}(t)=m_{i}(t)+\sum_{j \neq i} a_{j i}(t)$, where $m_{i}(t)$ is the death rate for the $i$ th population, so it is natural to impose $a_{i j}(t) \geq 0$ and $d_{i}(t)>\sum_{j \neq i} a_{j i}(t)$ for all $i, j$, i.e., $D(t)-A(t)^{T}$ is uniformly diagonally dominant for $t \geq 0$. It is also natural to assume that $a_{i j}(t)=\varepsilon_{i j}(t) a_{j i}(t)$ for $i \neq j$ and $t \geq 0$, with $\varepsilon_{i j}(t) \in(0,1]$, to account for some loss of the populations, when moving to different patches (see [27]), thus $[D(t)-A(t)] \mathbf{1} \gg 0$ for $t \geq 0$. If the mortality rates $m_{i}(t)$ are bounded below by a positive constant $m_{0}$, then $[D(t)-A(t)] \mathbf{1} \geq m_{0} \mathbf{1}$ for $t \geq 0$. To some degree, these comments justify assumption (h2) from a biological point of view.

Following the general approach in the literature, here multiple (time-varying) discrete delays have been introduced in the birth function. In biological terms, most situations do not require the consideration of more than one delay, either a discrete or a distributed delay, but occasionally multiple delays should be incorporated in each equation. For examples of such situations, we refer to generalizations of the classic Mackey-Glass model for the production of red blood cells in [1] and to [25] for other references.

As an important example of application, we have in mind the following non-autonomous Nicholson system with patch structure and multiple time-dependent discrete delays:

$$
x_{i}^{\prime}(t)=-d_{i}(t) x_{i}(t)+\sum_{j=1, j \neq i}^{n} a_{i j}(t) x_{j}(t)+\sum_{k=1}^{m} \beta_{i k}(t) x_{i}\left(t-\tau_{i k}(t)\right) e^{-c_{i k}(t) x_{i}\left(t-\tau_{i k}(t)\right)},
$$

for $i=1, \ldots, n, t \geq 0$. For (3.5), we shall always assume that the coefficient and delay functions satisfy (h1), (h3) and that $c_{i k}(t) \geq c_{i}>0$ are continuous and bounded. With nonlinearities given by $h_{i k}(t, x)=x e^{-c_{i k}(t) x}$ for all $i, k,(\mathrm{~h} 4)$ is obviously satisfied.

The autonomous version of (3.5) with $n=1$ and $m=1$ is the famous Nicholson's blowfly equation, given by $N^{\prime}(t)=-d N(t)+\beta N(t-\tau) e^{-a N(t-\tau)}(d, \beta, a, \tau>0)$. A large-scale literature on the scalar Nicholson's blowflies equation, on a number of generalizations and on related models has been produced since its introduction by Gurney et al. [13], and real world applications implemented. Nevertheless, a number of problems regarding scalar Nicholson-type equation still remain unsolved, see [2, 3] and references therein. On the other hand, results concerning multi-dimensional versions of such models are still quite limited. Not only is the literature on Nicholson systems very sparse, but also most authors have only treated autonomous Nicholson systems, and only recently have non-autonomous Nicholson systems been considered. See [4, 7, 10, 15, 16, 17, 28, 29, 31, also for biological details of the models and additional references. 
Besides Ricker-type nonlinearities as in the non-autonomous Nicholson system (3.5), other useful population models can be written in the form (3.1). Among them, are models with Mackey-Glass type nonlinearities of the form (see [19])

$$
h_{i k}(t, x)=x e^{-c_{i k}(t) x^{\alpha}} \quad(\alpha>0) \quad \text { or } \quad h_{i k}(t, x)=\frac{x}{1+c_{i k}(t) x^{\alpha}} \quad(\alpha \geq 1),
$$

which satisfy (h4) if $c_{i k}(t)$ are continuous and bounded below and above by positive constants.

System (3.1) is considered as a DDE in $C=C\left([-\tau, 0] ; \mathbb{R}^{n}\right)$, where $\tau=\max _{i, k} \sup _{t \geq 0} \tau_{i k}(t)$. Unless specifically mentioned, $\|\phi\|=\max _{\theta \in[-\tau, 0]}|\phi(\theta)|$ for $\phi \in C$, where $|\cdot|$ is the maximum norm in $\mathbb{R}^{n}$. Motivated by the applications to mathematical biology, only nonnegative solutions of (3.1) are meaningful. For this reason, initial conditions are taken in either $C^{+}$or $C_{0}$, where

$$
C_{0}=\left\{\varphi \in C^{+}: \varphi(0) \gg 0\right\} .
$$

Together with (3.1), we also consider its linearization at the origin:

$$
y_{i}^{\prime}(t)=-d_{i}(t) y_{i}(t)+\sum_{j=1, j \neq i}^{n} a_{i j}(t) y_{j}(t)+\sum_{k=1}^{m} \beta_{i k}(t) y_{i}\left(t-\tau_{i k}(t)\right), \quad i=1, \ldots, n .
$$

Write (3.1), (3.6) as $x^{\prime}(t)=f\left(t, x_{t}\right), y^{\prime}(t)=g\left(t, y_{t}\right)$ respectively, where $f=\left(f_{1}, \ldots, f_{n}\right), g=$ $\left(g_{1}, \ldots, g_{n}\right)$ and

$$
f_{i}(t, \phi)=-d_{i}(t) \phi_{i}(0)+\sum_{j=1, j \neq i}^{n} a_{i j}(t) \phi_{j}(0)+\sum_{k=1}^{m} \beta_{i k}(t) h_{i k}\left(t, \phi_{i}\left(-\tau_{i k}(t)\right)\right)
$$

and

$$
g_{i}(t, \phi)=-d_{i}(t) \phi_{i}(0)+\sum_{j=1, j \neq i}^{n} a_{i j}(t) \phi_{j}(0)+\sum_{k=1}^{m} \beta_{i k}(t) \phi_{i}\left(-\tau_{i k}(t)\right) .
$$

Assume (h1), (h3), (h4). For $t \geq 0$ and $\phi \geq 0, \phi_{i}(0)=0$, then $f_{i}(t, \phi) \geq 0$ and $g_{i}(t, \phi) \geq 0$, which implies that $x(t):=x(t, 0, \phi, f) \geq 0$ and $y(t):=y(t, 0, \phi, g) \geq 0$ for $t \geq 0$. Moreover, $x_{i}^{\prime}(t) \geq-d_{i}(t) x_{i}(t)$ and $y_{i}^{\prime}(t) \geq-d_{i}(t) y_{i}(t)$ for $t \geq 0$ and $1 \leq i \leq n$. Hence both $C^{+}$and $C_{0}$ are positively invariant for (3.1) and (3.6). The next result is a consequence of Theorem 2.3.

Theorem 3.1. Under the assumptions (h1)-(h4), all solutions of (3.1) with initial conditions in $C_{0}$ are defined and strictly positive on $[0, \infty)$; moreover, there exists $L>0$ such that, for any $\phi \in C_{0}$, there is $T=T(\phi)>0$ such that

$$
0<x_{i}(t, 0, \phi)<L \quad \text { for } \quad t \geq T, i=1, \ldots, n .
$$

We now introduce a notation often used for DDEs (cf. [24], p. 82): if there is no possibility of misinterpretation with intervals of $\mathbb{R}$ or $\mathbb{R}^{n}$, for $v \in \mathbb{R}^{n}$ we also denote $[0, v]$ and $[v, \infty)$ as the subsets of $C$ given by $[0, v]=\left\{v \in C^{+}: \varphi \leq v\right\}$ and $[v, \infty)=\{v \in C: \varphi \geq v\}$. 
Lemma 3.1. Under (h1), (h3), system (3.6) is cooperative, and the following holds:

(i) If there exist a vector $v=\left(v_{1}, \ldots, v_{n}\right) \gg 0$ and $T_{0} \geq 0$ such that $M(t) v \leq 0$ for $t \geq T_{0}$, then the sets $[0, c v] \cap C_{0}$ (where $c>0$ ) are invariant for (3.6) with $t \geq T_{0}$; in particular, the solutions of (3.6) are uniformly stable.

(ii) If there exist a vector $v=\left(v_{1}, \ldots, v_{n}\right) \gg 0$ and $T_{0} \geq 0$ such that $M(t) v \geq 0$ for $t \geq T_{0}$, then the sets $[c v, \infty) \cap C_{0}$ (where $c>0$ ) are invariant for (3.6) with $t \geq T_{0}$.

Proof. Since $g$ satisfies (Q), (3.6) is cooperative. Let $M(t) v \leq 0$ for $t \geq T_{0}$, for some strictly positive vector $v=\left(v_{1}, \ldots, v_{n}\right) \in \mathbb{R}^{n}$ and some $T_{0} \geq 0$. For $\phi \in C^{+}$with $\phi \leq v$, if $\phi_{i}(0)=v_{i}$ for some $i$, then $g_{i}(t, \phi) \leq(M(t) v)_{i} \leq 0$ for $t \geq T_{0}$, proving that $[0, v] \cap C_{0}$ is positively invariant (see Lemma [2.3); since the system is linear, for any positive constant $c$ the set $[0, c v] \cap C_{0}$ is positively invariant as well. From the monotonicity, it follows that the solution $y=0$ of (3.6) is uniformly stable. The proof of (ii) is similar.

Definition 3.1. The trivial solution $x \equiv 0$ of (3.1) is said to be stable if for any $\varepsilon>0$ there is $\delta=\delta(\varepsilon)>0$ such that $\left\|x_{t}(0, \phi)\right\|<\varepsilon$ for all $\phi \in C_{0}$ with $\|\phi\|<\delta$ and $t \geq 0 ; 0$ is said to be globally attractive (in $C_{0}$ ) if $x(t, 0, \phi) \rightarrow 0$ as $t \rightarrow \infty$, for all solutions of (3.1) with initial conditions $x_{0}=\phi \in C_{0} ; 0$ is globally asymptotically stable (GAS) if it is stable and globally attractive.

The next result gives sufficient conditions for the stability and global attractivity of the trivial equilibrium. When (3.1) refers to a population model, the global attractivity of 0 means the extinction of the populations in all patches.

Theorem 3.2. Assume (h1), (h3) and (h4) with $0<h_{i}^{+}(x)<x, x>0,1 \leq i \leq n$. Further suppose that:

(i) there exist $v=\left(v_{1}, \ldots, v_{n}\right) \gg 0$ and $T_{0} \geq 0$ such that $M(t) v \leq 0$ for $t \geq T_{0}$;

(ii) either $\liminf \operatorname{int}_{t \rightarrow \infty} \beta_{i}(t)>0$ or $\limsup _{t \rightarrow \infty}(M(t) v)_{i}<0$, for all $i=1, \ldots, n$.

Then the trivial solution of (3.1) is GAS in $C_{0}$.

Proof. From (ii), for each $i=1, \ldots, n$, either $\beta_{i}(t) \geq \beta_{i}>0$ for $t$ large or $(M(t) v)_{i} \leq-\lambda_{i}<0$ for $t$ large. In particular, together with (h3), conditions (i) and (ii) imply (h2).

For $\phi \in C_{0}, t_{0} \geq 0$ and $i \in\{1, \ldots, n\}$, it holds $f_{i}(t, \phi) \leq g_{i}(t, \phi)$. In this way, the solutions of (3.1) and (3.6) satisfy $x\left(t, t_{0}, \phi, f\right) \leq y\left(t, t_{0}, \phi, g\right), t \geq t_{0}$. From Lemma 3.1, the zero solution of (3.1) is stable. Now, we show that it attracts all solutions with initial conditions in $C_{0}$.

With $\hat{x}_{j}(t)=x_{j}(t) / v_{j}$, system (3.1) reads as

$$
\hat{x}_{i}^{\prime}(t)=-d_{i}(t) \hat{x}_{i}(t)+\sum_{j=1, j \neq i}^{n} \hat{a}_{i j}(t) \hat{x}_{j}(t)+\sum_{k=1}^{m} \beta_{i k}(t) \hat{h}_{i k}\left(t, \hat{x}_{i}\left(t-\tau_{i k}(t)\right)\right), i=1, \ldots, n, t \geq 0,
$$

where $\hat{a}_{i j}(t)=v_{i}^{-1} a_{i j}(t) v_{j}, j \neq i$, and $\hat{h}_{i k}(t, x)=v_{i}^{-1} h_{i k}\left(t, v_{i} x\right)$ satisfy (h4). Hence, without loss of generality we consider the original system (3.1) and take $v=\mathbf{1}$ in (i), (ii).

The solutions $x(t)=x\left(t, t_{0}, \phi, f\right)$ are bounded, so define $u_{j}=\limsup _{t \rightarrow \infty} x_{j}(t)$ and let $u_{i}=$ $\max _{1 \leq j \leq n} u_{j}$. If $u_{i}>0$, by the fluctuation lemma take a sequence $\left(t_{k}\right)$ with $t_{k} \rightarrow \infty, x_{i}\left(t_{k}\right) \rightarrow$ 
$u_{i}, x_{i}^{\prime}\left(t_{k}\right) \rightarrow 0$. For any small $\varepsilon>0$ with $u_{i}-\varepsilon>0$, for $k$ large we get $t_{k} \geq T_{0}+\tau, u_{j}-\varepsilon \leq x_{j}(t) \leq u_{j}+\varepsilon$ and $h_{i k}\left(t, x_{i}(t)\right) \leq \max _{x \in\left[0, u_{i}+\varepsilon\right]} h_{i}^{+}(x)$, for $t \in\left[t_{k}-\tau, t_{k}\right]$. Thus,

$$
\begin{aligned}
x_{i}^{\prime}\left(t_{k}\right) & \leq-d_{i}\left(t_{k}\right)\left(u_{i}-\varepsilon\right)+\sum_{j \neq i} a_{i j}\left(t_{k}\right)\left(u_{j}+\varepsilon\right)+\sum_{p} \beta_{i p}\left(t_{k}\right) h_{i}^{+}\left(x_{i}\left(t_{k}-\tau_{i p}\left(t_{k}\right)\right)\right) \\
& \leq-u_{i}\left(d_{i}\left(t_{k}\right)-\sum_{j \neq i} a_{i j}\left(t_{k}\right)\right)+\beta_{i}\left(t_{k}\right) \max _{x \in\left[0, u_{i}+\varepsilon\right]} h_{i}^{+}(x)+O(\varepsilon) .
\end{aligned}
$$

Taking limits $k \rightarrow \infty, \varepsilon \rightarrow 0^{+}$, we derive that

$$
0 \leq \limsup _{t \rightarrow \infty} u_{i}\left(\beta_{i}(t)-d_{i}(t)+\sum_{j \neq i} a_{i j}(t)\right)+\left(\max _{x \in\left[0, u_{i}\right]} h_{i}^{+}(x)-u_{i}\right) \liminf _{t \rightarrow \infty} \beta_{i}(t) .
$$

Since $\max _{x \in\left[0, u_{i}\right]} h_{i}^{+}(x)<u_{i}$ and one of the conditions in (ii) is satisfied, this is not possible. Therefore $u_{i}=0$, and the proof is complete.

For the definitions of persistence and permanence given below, see e.g. [26].

Definition 3.2. A set $S \subset C^{+}$is an admissible set of initial conditions for $x^{\prime}(t)=f\left(t, x_{t}\right)$ if any solution $x(t, 0, \phi)$ with initial condition $x_{0}=\phi \in S$ satisfies $x_{t} \in S$ for $t \geq 0$, whenever it is defined. A DDE $x^{\prime}(t)=f\left(t, x_{t}\right)$ is said to be persistent in $S$, for $S$ an admissible set of initial conditions, if all solutions $x(t, 0, \phi)$ with $\phi \in S$ are defined and bounded below away from zero on $[0, \infty)$, i.e., $\liminf _{t \rightarrow \infty} x_{i}(t, 0, \phi)>0$ for all $1 \leq i \leq n, \phi \in S$; and $x^{\prime}(t)=f\left(t, x_{t}\right)$ is uniformly persistent in $S$ if there is $m>0$ such that $\liminf _{t \rightarrow \infty} x_{i}(t, 0, \phi) \geq m$ for all $1 \leq i \leq n, \phi \in S$. The system is said to be permanent in $S$ if it is dissipative and uniformly persistent; in other words, all solutions $x(t, 0, \phi), \phi \in S$, are defined on $[0, \infty)$ and there are positive constants $m, M$ such that, given any $\phi \in S$, there exists $t_{0}=t_{0}(\phi)$ for which

$$
m \leq x_{i}(t, 0, \phi) \leq M, \quad 1 \leq i \leq n, t \geq t_{0} .
$$

Hereafter, unless otherwise stated, the notions of persistence, uniform persistence and permanence always refer to the choice of $S=C_{0}$ as the set of admissible initial conditions.

Observe that a linear homogeneous DDE system is uniformly persistent (in $C_{0}$ ) if and only if all components of all solutions with initial conditions in $C_{0}$ tend to $\infty$ as $t \rightarrow \infty$. The next result concerns the uniform persistence of (3.6).

Proposition 3.1. Assume (h1), (h3), and that there exist vectors $v \gg 0, \eta \gg 0$ such that

$$
M(t) v \geq \eta \quad \text { for large } t>0 .
$$

Then all solutions $y(t)$ of (3.6) with initial conditions in $C_{0}$ satisfy $\lim _{t \rightarrow \infty} y_{i}(t)=\infty, i=1, \ldots, n$.

Proof. For $\phi \in C_{0}, t_{0} \geq \tau$, we have $y_{t}=y_{t}\left(t_{0}, \phi\right) \in$ int $C^{+}$for $t \geq t_{0}$, thus $y_{\tau} \geq c v$ for some small $c>0$. System (3.6) is linear and cooperative, with $[v, \infty)$ forward invariant for $t$ on the interval $\left[T_{0}, \infty\right)$ if $M(t) v \geq 0$ for $t \geq T_{0}$. To simplify the exposition, as before we take $v=\mathbf{1}$. We only need to show that all components $u_{i}(t)$ of the solution $u(t):=y\left(t, T_{0}, \mathbf{1}\right)$ satisfy $\lim _{t \rightarrow \infty} u_{i}(t)=\infty$. 
For $j \in\{1, \ldots, n\}$, let $c_{j}=\liminf _{t \rightarrow \infty} x_{j}(t) \in[1, \infty]$. Suppose that $c_{j}<\infty$ for some $j \in$ $\{1, \ldots, n\}$, and take $c_{i}=\min _{j} c_{j}$, for the natural ordering in $(0, \infty]$. Then, there is a sequence $t_{k} \rightarrow \infty$ such that $u_{i}\left(t_{k}\right) \rightarrow c_{i}, u_{i}^{\prime}\left(t_{k}\right) \rightarrow 0$. On the other hand, from (3.9) there are $\eta_{i}, T_{1}>0$ such that $\beta_{i}(t)-d_{i}(t)+\sum_{j \neq i} a_{i j}(t) \geq \eta_{i}>0, t \geq T_{1}$. For any small $\varepsilon>0$ and $k$ sufficiently large, we obtain

$$
\begin{aligned}
u_{i}^{\prime}\left(t_{k}\right) & \geq-d_{i}\left(t_{k}\right) u_{i}\left(t_{k}\right)+\left(c_{i}-\varepsilon\right)\left(\sum_{j \neq i} a_{i j}\left(t_{k}\right)+\beta_{i}\left(t_{k}\right)\right) \\
& \geq d_{i}\left(t_{k}\right)\left[-u_{i}\left(t_{k}\right)+\left(c_{i}-\varepsilon\right)\right]+\left(c_{i}-\varepsilon\right) \eta_{i}
\end{aligned}
$$

and therefore $0 \geq c_{i} \eta_{i}>0$, which is not possible. This ends the proof.

For dissipative systems (3.1) with nonlinearities satisfying (h4), the above criterion for the uniform persistence of the linearization at zero also provides a criterion for its uniform persistence. This is stated in the main theorem of this section, given below. For a relevant extension, see Theorem 3.4 ,

Theorem 3.3. Assume (h1)-(h4), and suppose that there exist $v \gg 0, \eta \gg 0$ such that (3.9) is satisfied. Then (3.1) is uniformly persistent, and thus permanent.

Proof. After effecting a scaling of the variables, we take $v=(1, \ldots, 1)=1$ in condition (3.9), thus there exist constants $\eta_{i}>0(i=1, \ldots, n)$ such that, for some $T_{0}$,

$$
\beta_{i}(t) \geq d_{i}(t)-\sum_{j \neq i} a_{i j}(t)+\eta_{i}, \quad t \geq T_{0}
$$

On the other hand, $d_{i}(t)-\sum_{j \neq i} a_{i j}(t) \leq \bar{d}_{i}:=\sup _{t \geq T_{0}} d_{i}(t)$, and with $1<\alpha_{i}<1+\eta_{i} / \bar{d}_{i}$ we obtain

$$
\alpha_{i}^{-1} \beta_{i}(t)-d_{i}(t)+\sum_{j \neq i} a_{i j}(t)>0, \quad \text { for } \quad t \geq T_{0}, i=1, \ldots, n .
$$

For $h_{i}^{-}$as in (h4), we can choose $L>m>0$ such that the uniform estimate (3.7) holds, $h_{i}^{-}(m)=\min _{x \in[m, L]} h_{i}^{-}(x)$, with $\left(h_{i}^{-}\right)^{\prime}(x)>0$ and $\alpha_{i}^{-1} x<h_{i}^{-}(x)$ for $x \in(0, m]$ and all $i$.

Consider the auxiliary cooperative system

$$
\begin{aligned}
x_{i}^{\prime}(t) & =-d_{i}(t) x_{i}(t)+\sum_{j=1, j \neq i}^{n} a_{i j}(t) x_{j}(t)+\sum_{k=1}^{m} \beta_{i k}(t) H_{i}\left(x_{i}\left(t-\tau_{i k}(t)\right)\right) \\
& =: F_{i}\left(t, x_{t}\right), \quad i=1, \ldots, n, t \geq 0,
\end{aligned}
$$

where $H_{i}(x)=h_{i}^{-}(x)$ if $0 \leq x \leq m, H_{i}(x)=h_{i}^{-}(m)$ if $x \geq m$.

For $x(t)$ a positive solution of (3.1), for $t>0$ sufficiently large and $1 \leq i \leq n$, we have $x_{i}(t) \leq L$ and $h_{i k}\left(t, x_{i}\left(t-\tau_{i k}(t)\right)\right) \geq H_{i}\left(x_{i}\left(t-\tau_{i k}(t)\right)\right)$. Therefore, if (3.11) is uniformly persistent, then (3.1) is uniformly persistent as well.

Now, we consider any solution $x(t)=x\left(t, T_{0}, \phi, F\right)$ of (3.11) with $x_{T_{0}}=\phi \in C_{0}$ (where $T_{0}$ is as in (3.10) $)$, and claim that

$$
\liminf _{t \rightarrow \infty} x_{i}(t) \geq m, \quad 1 \leq i \leq n
$$


In fact, we shall show that there exists $T \geq 0$ such that

$$
x_{i}(t) \geq m \quad \text { for } \quad t \geq T, 1 \leq i \leq n .
$$

The proof, inspired by some arguments in [10], is divided into several steps.

Step 1. We prove that if $\min \left\{x_{j}(t): 1 \leq j \leq n, t \in[T, T+\tau]\right\} \geq m$ for some $T \geq T_{0}$, then $x_{j}(t) \geq m$ for all $t \geq T$ and $j=1, \ldots, n$.

Assume that $x_{j}(t) \geq m$ for $t \in[T, T+\tau]$ and $j=1, \ldots, n$. Let $t_{0} \in[T+\tau, T+2 \tau]$ and $i \in\{1, \ldots, n\}$ such that $x_{i}\left(t_{0}\right)=\min \left\{x_{j}(t): 1 \leq j \leq n, t \in[T+\tau, T+2 \tau]\right\}$.

If $x_{i}\left(t_{0}\right)<m$, we have

$$
0 \geq x_{i}^{\prime}\left(t_{0}\right)=-d_{i}\left(t_{0}\right) x_{i}\left(t_{0}\right)+\sum_{j=1, j \neq i}^{n} a_{i j}\left(t_{0}\right) x_{j}\left(t_{0}\right)+\sum_{k=1}^{m} \beta_{i k}(t) H_{i}\left(x_{i}\left(t_{0}-\tau_{i k}\left(t_{0}\right)\right)\right) .
$$

Note that $x_{i}\left(t_{0}-\tau_{i k}\left(t_{0}\right)\right) \geq m$ if $t_{0}-\tau_{i k}\left(t_{0}\right) \in[T, T+\tau]$ and $x_{i}\left(t_{0}-\tau_{i k}\left(t_{0}\right)\right) \geq x_{i}\left(t_{0}\right)$ if $t_{0}-\tau_{i k}\left(t_{0}\right) \in$ $\left[T+\tau, t_{0}\right]$, hence $H_{i}\left(x_{i}\left(t_{0}-\tau_{i k}\left(t_{0}\right)\right)\right) \geq H_{i}\left(x_{i}\left(t_{0}\right)\right)$. From (3.10) and the definition of $m$ we obtain

$$
\begin{aligned}
0 & \geq\left(-d_{i}\left(t_{0}\right)+\sum_{j=1}^{n} a_{i j}\left(t_{0}\right)\right) x_{i}\left(t_{0}\right)+\beta_{i}\left(t_{0}\right) H_{i}\left(x_{i}\left(t_{0}\right)\right) \\
& \geq\left(-d_{i}\left(t_{0}\right)+\sum_{j=1}^{n} a_{i j}\left(t_{0}\right)+\alpha_{i}^{-1} \beta_{i}\left(t_{0}\right)\right) x_{i}\left(t_{0}\right)>0,
\end{aligned}
$$

which is not possible. Thus, $x_{i}\left(t_{0}\right) \geq m$. By iteration, this proves Step 1 .

Step 2. Next, for any $T_{0}$ as in (3.10) and $s_{0}:=\min \left\{x_{j}(t): 1 \leq j \leq n, t \in\left[T_{0}, T_{0}+\tau\right]\right\}$, we shall show the estimate

$$
\min _{j} \min _{t \in\left[T_{0}+\tau, T_{0}+2 \tau\right]} x_{j}(t) \geq s_{1}
$$

where

$$
s_{1}:=\min \left\{m, \min _{j}\left(\alpha_{j} H_{j}\left(s_{0}\right)\right)\right\} .
$$

To simplify the exposition, take $T_{0}=0$. In this way, we denote $s_{0}:=\min \left\{x_{j}(t): 1 \leq j \leq n, t \in\right.$ $[0, \tau]\}>0$. If $s_{0} \geq m$, from Step 1 the proof is complete. Now, consider the case $s_{0}<m$. By the definition of $m, h_{j}^{-}\left(s_{0}\right) \alpha_{j}=H_{j}\left(s_{0}\right) \alpha_{j}>s_{0}$ for all $j$, thus $s_{1}>s_{0}$. We claim that

$$
\min _{j} \min _{t \in[\tau, 2 \tau]} x_{j}(t) \geq s_{1}
$$

Otherwise, there are $t_{1} \in[\tau, 2 \tau]$ and $i \in\{1, \ldots, n\}$ such that $x_{i}\left(t_{1}\right)<s_{1}$ and $x_{j}(t) \geq x_{i}\left(t_{1}\right)$ for all $t \in\left[\tau, t_{1}\right]$ and $j \in\{1, \ldots, n\}$.

Since $x_{i}\left(t_{1}-\tau_{i k}\left(t_{1}\right)\right) \geq \min \left\{s_{0}, x_{i}\left(t_{1}\right)\right\}$, we have $H_{i}\left(x_{i}\left(t_{1}-\tau_{i k}\left(t_{1}\right)\right)\right) \geq \min \left\{H_{i}\left(s_{0}\right), H_{i}\left(x_{i}\left(t_{1}\right)\right)\right\}$. We now consider two cases separately.

If $s_{0} \geq x_{i}\left(t_{1}\right)$, then $H_{i}\left(s_{0}\right) \geq H_{i}\left(x_{i}\left(t_{1}\right)\right)$ and we get (3.13) with $t_{0}$ replaced by $t_{1}$, thus a contradiction. 
If $s_{0}<x_{i}\left(t_{1}\right)$, then $H_{i}\left(s_{0}\right)<H_{i}\left(x_{i}\left(t_{1}\right)\right)$. Since $x_{i}\left(t_{1}\right)<s_{1} \leq \alpha_{i} H_{i}\left(s_{0}\right)$, we derive

$$
\begin{aligned}
0 \geq x_{i}^{\prime}\left(t_{1}\right) & \geq\left(-d_{i}\left(t_{1}\right)+\sum_{j=1}^{n} a_{i j}\left(t_{1}\right)\right) x_{i}\left(t_{1}\right)+\beta_{i}\left(t_{1}\right) H_{i}\left(s_{0}\right) \\
& >\left(-d_{i}\left(t_{1}\right)+\sum_{j=1}^{n} a_{i j}\left(t_{1}\right)+\beta_{i}\left(t_{1}\right) \alpha_{i}^{-1}\right) x_{i}\left(t_{1}\right)>0,
\end{aligned}
$$

which is again a contradiction. This proves the estimate (3.14).

Step 3. Now, we define by recurrence the sequence

$$
s_{k+1}=\min \left\{m, \min _{j}\left(\alpha_{j} H_{j}\left(s_{k}\right)\right)\right\}, \quad k \in \mathbb{N}_{0} .
$$

If $s_{k}=m$ for some $k \in \mathbb{N}$, (3.12) follows by Steps 1 and 2. In this case, $\alpha_{j} H_{j}\left(s_{k}\right)>m$, hence $s_{p}=m$ for all $p>k$. If $s_{k}<m$ for all $k,\left(s_{k}\right)$ is strictly increasing, because

$$
s_{k+1}=\min _{j}\left(\alpha_{j} H_{j}\left(s_{k}\right)\right)>s_{k}
$$

For $s^{*}=\lim s_{k}$, from the definition of $m$ we derive

$$
0<s^{*} \leq m \text { and } \quad s^{*} \geq \min _{j} \alpha_{j} H_{j}\left(s^{*}\right)>s^{*},
$$

which is not possible. The proof is complete.

Remark 3.2. We observe that assumptions (h2) and (3.9) are satisfied if $\liminf _{t \rightarrow \infty} \beta_{i}(t)>0$ and

$$
\gamma_{i} \geq \frac{\beta_{i}(t) v_{i}}{d_{i}(t) v_{i}-\sum_{j \neq i} a_{i j}(t) v_{j}} \geq \alpha_{i}>1, \quad \text { for } \quad t \geq T_{0}, i=1, \ldots, n,
$$

for some vector $v=\left(v_{1}, \ldots, v_{n}\right) \gg 0$ and constants $\alpha_{i}, \gamma_{i}$.

Example 3.1. Consider the system

$$
x_{i}^{\prime}(t)=-d_{i}(t) x_{i}(t)+\sum_{j=1, j \neq i}^{n} a_{i j}(t) x_{j}(t)+\beta_{i}(t) \frac{\left.x_{i}\left(t-\tau_{i}(t)\right)\right)}{1+c_{i}(t) x_{i}^{\alpha_{i}}\left(t-\tau_{i}(t)\right)}, i=1, \ldots, n, t \geq 0,
$$

where $\alpha_{i} \geq 1, d_{i}(t)>0, a_{i j}(t) \geq 0, c_{i}(t), \beta_{i}(t), \tau_{i}(t) \geq 0$ are continuous and bounded and $0<c_{i}^{-} \leq$ $c_{i}(t) \leq c_{i}^{+}, \beta_{i}(t) \geq \beta_{i}^{-}>0, t \geq 0, i=1, \ldots, n$. For $h_{i}(t, x)=x\left(1+c_{i}(t) x^{\alpha_{i}}\right)^{-1}, h_{i}^{ \pm}(x)=x(1+$ $\left.c_{i}^{\mp} x^{\alpha_{i}}\right)^{-1}$, we have $h_{i}^{-}(x) \leq h_{i}(t, x) \leq h_{i}^{+}(x), h_{i}^{ \pm}(0)=0,\left(h_{i}^{ \pm}\right)^{\prime}(0)=1$ and $0<h_{i}^{-}(x) \leq h_{i}^{+}(x)<x$ for $x>0$. For each vector $v=\left(v_{1}, \ldots, v_{n}\right) \gg 0$, define

$$
l_{i}(v)=\liminf _{t \rightarrow \infty} \frac{\beta_{i}(t) v_{i}}{d_{i}(t) v_{i}-\sum_{j \neq i} a_{i j}(t) v_{j}}, L_{i}(v)=\limsup _{t \rightarrow \infty} \frac{\beta_{i}(t) v_{i}}{d_{i}(t) v_{i}-\sum_{j \neq i} a_{i j}(t) v_{j}}, \quad i=1, \ldots, n .
$$

From Theorem 3.2 and Remark 3.2, the zero solution of (3.16) is GAS if there exists $v \gg 0$ such that $L_{i}(v)<1$ for all $i$, whereas (3.16) is permanent if there exists $v \gg 0$ such that $1<l_{i}(v), L_{i}(v)<\infty$ for all $i$. 
A careful reading of the proof above leads to several generalizations. First, it is clear that, in the statement of Theorem 3.3. hypothesis (h2) can actually be replaced by the dissipativeness of the system. Having this in mind, one also sees that the same arguments apply to dissipative systems more general than (3.1), where, in each equation $i$, the instantaneous terms $a_{i j}(t) x_{j}(t)$ are replaced by linear delayed terms and the nonlinear terms are as in (3.3). This is expressed in the next theorem.

Theorem 3.4. Consider a non-autonomous system of one of the forms

$$
\begin{aligned}
x_{i}^{\prime}(t) & =-d_{i}(t) x_{i}(t)+\sum_{j=1, j \neq i}^{n} a_{i j}(t) \int_{-\tau}^{0} x_{j}(t+s) d_{s} \nu_{i j}(t, s) \\
& +\sum_{k=1}^{m} \beta_{i k}(t) \int_{-\tau}^{0} h_{i k}\left(s, x_{i}(t+s)\right) d_{s} \eta_{i k}(t, s), i=1, \ldots, n, t \geq 0, \\
x_{i}^{\prime}(t) & =-d_{i}(t) x_{i}(t)+\sum_{j=1, j \neq i}^{n} a_{i j}(t) \int_{-\tau}^{0} x_{j}(t+s) d_{s} \nu_{i j}(t, s) \\
& +\sum_{k=1}^{m} \beta_{i k}(t) h_{i k}\left(t, \int_{-\tau}^{0} x_{i}(t+s) d_{s} \eta_{i k}(t, s)\right), i=1, \ldots, n, t \geq 0,
\end{aligned}
$$

where: $d_{i}(t), a_{i j}(t), \beta_{i k}(t), h_{i k}(t, x)$ satisfy (h1), (h3) and (h4); the measurable functions $\nu_{i j}, \eta_{i k}$ : $[0, \infty) \times[-\tau, 0] \rightarrow \mathbb{R}$ are continuous from the left in $s, \nu_{i j}(t, \cdot), \eta_{i k}(t, \cdot)$ are non-decreasing and normalized so that

$$
\int_{-\tau}^{0} d_{s} \nu_{i j}(t, s)=\int_{-\tau}^{0} d_{s} \eta_{i k}(t, s)=1, \quad i, j=1, \ldots, n, k=1, \ldots, m, t \geq 0 .
$$

Assume also that (3.17), or (3.18), is dissipative and that (3.9) is satisfied for some $v, \eta \gg 0$. Then, the system is uniformly persistent.

We now apply the previous theorems to the Nicholson system (3.5).

Theorem 3.5. Consider system (3.5), where $d_{i}, a_{i j}, \beta_{i k}, c_{i k}, \tau_{i k}:[0, \infty) \rightarrow[0, \infty)$ are continuous and bounded, with $d_{i}(t), \beta_{i}(t)=\sum_{k=1}^{m} \beta_{i k}(t)$ strictly positive and $c_{i k}(t) \geq c_{i}>0$ on $[0, \infty)$, for all $i, j, k$. With the notation in (3.4), assume that there exist a vector $u \gg 0$ and $\delta>0, T_{0} \geq 0$ such that $[D(t)-A(t)] u \geq \delta \mathbf{1}$ for $t \geq T_{0}$. Then:

(i) Eq. (3.5) is dissipative.

(ii) If there exist a vector $v \gg 0$ and $T_{0} \geq 0$ such that $M(t) v \leq 0$ for $t \geq T_{0}$ and either $\lim \inf _{t \rightarrow \infty} \beta_{i}(t) \gg 0$ or $\lim \sup _{t \rightarrow \infty}(M(t) v)_{i} \ll 0(1 \leq i \leq n)$, the zero solution of (3.5) is GAS.

(iii) If there exist vectors $v \gg 0, \eta \gg 0$ such that $M(t) v \geq \eta$ for $t \geq T_{0}$, then (3.5) is permanent.

Example 3.2. Consider the planar system

$$
\begin{aligned}
& x_{1}^{\prime}(t)=-\left(1+\cos ^{2} t\right) x_{1}(t)+\gamma_{1}\left(1+\sin ^{2} t\right) x_{2}(t)+\sum_{j=1}^{m}\left(\beta_{1 j}+f_{1 j}(t)\right) x_{1}\left(t-\tau_{1 j}(t)\right) e^{-c_{1 j}(t) x_{1}\left(t-\tau_{1 j}(t)\right)}, \\
& x_{2}^{\prime}(t)=-\left(1+\sin ^{2} t\right) x_{2}(t)+\gamma_{2}\left(1+\cos ^{2} t\right) x_{1}(t)+\sum_{j=1}^{m}\left(\beta_{2 j}+f_{2 j}(t)\right) x_{2}\left(t-\tau_{2 j}(t)\right) e^{-c_{2 j}(t) x_{2}\left(t-\tau_{2 j}(t)\right)},
\end{aligned}
$$


where $\gamma_{i}>0, \beta_{i j} \geq 0$ with $\beta_{i}:=\sum_{j=1}^{m} \beta_{i j}>0$, all the functions $f_{i j}(t), c_{i j}(t), \tau_{i j}(t)$ are continuous, nonnegative and bounded on $[0, \infty)$, with $c_{i j}(t)$ bounded below by positive constants, for $t \geq 0, i=$ $1,2, j=1, \ldots, m$. Write $f_{i}(t):=\sum_{j=1}^{m} f_{i j}(t)$, let $f_{i}^{-}, f_{i}^{+}$be such that $0 \leq f_{i}^{-} \leq f_{i}(t) \leq f_{i}^{+}$for $t \geq 0$ and denote $\beta_{i}^{-}=\beta_{i}+f_{i}^{-}, \beta_{i}^{+}=\beta_{i}+f_{i}^{+}, i=1,2$. With the notation in (3.4), we have

$$
\begin{aligned}
D(t)-A(t) & =\left[\begin{array}{cc}
1+\cos ^{2} t & -\gamma_{1}\left(1+\sin ^{2} t\right) \\
-\gamma_{2}\left(1+\cos ^{2} t\right) & 1+\sin ^{2} t
\end{array}\right], \\
M(t) & =\left[\begin{array}{cc}
\beta_{1}+f_{1}(t)-\left(1+\cos ^{2} t\right) & \gamma_{1}\left(1+\sin ^{2} t\right) \\
\gamma_{2}\left(1+\cos ^{2} t\right) & \beta_{2}+f_{2}(t)-\left(1+\sin ^{2} t\right)
\end{array}\right] .
\end{aligned}
$$

Consider a vector $u=\left(1, u_{2}\right)$ with $u_{2}>0$, and write $[D(t)-A(t)] u=\left[\begin{array}{l}\delta_{1}(t) \\ \delta_{2}(t)\end{array}\right]$. Since min $\delta_{1}(t)=$ $1-2 u_{2} \gamma_{1}, \min \delta_{2}(t)=u_{2}-2 \gamma_{2}$, if

$$
4 \gamma_{1} \gamma_{2} \leq 1
$$

we can find $u_{2}$ such that $2 \gamma_{2} \leq u_{2} \leq\left(2 \gamma_{1}\right)^{-1}$, implying that $[D(t)-A(t)] u \geq 0$ for $t \in \mathbb{R}$. On the other hand, $\delta_{1}\left(\frac{\pi}{4}\right)=\frac{3}{2}\left(1-u_{2} \gamma_{1}\right)>0, \delta_{2}\left(\frac{\pi}{4}\right)=\frac{3}{2}\left(-\gamma_{2}+u_{2}\right)>0$. By Theorem 2.1, we conclude that the ODE $x^{\prime}=-[D(t)-A(t)] x$ is globally exponentially stable.

We now look for a vector $v=\left(1, v_{2}\right) \gg 0$ such that $M(t) v \geq \eta \gg 0$. Write $M(t) v=\left[\begin{array}{l}m_{1}(t) \\ m_{2}(t)\end{array}\right]$ and observe that $m_{1}(t) \geq \eta_{1}:=\beta_{1}^{-}-2+v_{2} \gamma_{1}, m_{2}(t) \geq \eta_{2}:=v_{2}\left(\beta_{2}^{-}-2\right)+\gamma_{2}, t \geq 0$. Now assume that:

$$
\text { either } \beta_{1}^{-} \geq 2 \quad \text { or } \quad \beta_{2}^{-} \geq 2 \quad \text { or } \quad\left(2-\beta_{1}^{-}\right)\left(2-\beta_{2}^{-}\right)<\gamma_{1} \gamma_{2} \text {. }
$$

One easily verifies that: (i) if either $\beta_{1}^{-} \geq 2$ or $\beta_{2}^{-} \geq 2$, one can find $v_{2}>0$ such that $\eta_{1}>0, \eta_{2}>0$; (ii) if $\beta_{i}^{-}<2$ for $i=1,2$, and $\left(2-\beta_{1}^{-}\right)\left(2-\beta_{2}^{-}\right)<\gamma_{1} \gamma_{2}$, for any $v_{2}$ such that $\left(2-\beta_{1}\right) \gamma_{1}^{-1}<v_{2}<$ $\gamma_{2}\left(2-\beta_{2}\right)^{-1}$ we have $M(t) v \geq \eta=\left(\eta_{1}, \eta_{2}\right) \gg 0$. From Theorem 3.5.(iii), conditions (3.20)-(3.21) imply that (3.19) is permanent.

As an illustration, with $m=1$ and $\beta_{1}=2, \gamma_{1}=1=4 \gamma_{2}, f_{1}(t)=f_{2}(t)=0$, we conclude that

$$
\begin{aligned}
& x_{1}^{\prime}(t)=-\left(1+\cos ^{2} t\right) x_{1}(t)+\left(1+\sin ^{2} t\right) x_{2}(t)+2 x_{1}\left(t-\tau_{1 j}(t)\right) e^{-c_{1 j}(t) x_{1}\left(t-\tau_{1 j}(t)\right)} \\
& x_{2}^{\prime}(t)=-\left(1+\sin ^{2} t\right) x_{2}(t)+\frac{1}{4}\left(1+\cos ^{2} t\right) x_{1}(t)+\beta_{2} x_{2}\left(t-\tau_{2 j}(t)\right) e^{-c_{2 j}(t) x_{2}\left(t-\tau_{2 j}(t)\right)}
\end{aligned}
$$

is permanent for any $\beta_{2}>0$.

On reverse, if $\beta_{i}^{+}<1, i=1,2$, for a positive vector $v=\left(1, v_{2}\right)$ we obtain $M(t) v=\left[\begin{array}{l}m_{1}(t) \\ m_{2}(t)\end{array}\right]$ with $m_{1}(t) \leq \eta_{1}:=\beta_{1}^{+}-1+2 v_{2} \gamma_{1}, m_{2}(t) \leq \eta_{2}:=v_{2}\left(\beta_{2}^{+}-1\right)+2 \gamma_{2}, t \geq 0$. At this point, assume

$$
\beta_{i}^{+}<1, i=1,2 \text { and } 4 \gamma_{1} \gamma_{2} \leq\left(1-\beta_{1}^{+}\right)\left(1-\beta_{2}^{+}\right) .
$$

Thus, choosing $v_{2}$ such that $2 \gamma_{2}\left(1-\beta_{2}^{+}\right)^{-1} \leq v_{2} \leq\left(2 \gamma_{1}\right)^{-1}\left(1-\beta_{1}^{+}\right)$we obtain $M(t) v \leq 0$ for all $t \geq 0$. From Theorem 3.5.(ii), conditions (3.23) imply that the trivial solution of (3.19) is GAS. In particular, this is the case of the zero solution of

$$
\begin{aligned}
& x_{1}^{\prime}(t)=-\left(1+\cos ^{2} t\right) x_{1}(t)+\frac{1}{4}\left(1+\sin ^{2} t\right) x_{2}(t)+\frac{1}{2} x_{1}\left(t-\tau_{1 j}(t)\right) e^{-c_{1 j}(t) x_{1}\left(t-\tau_{1 j}(t)\right)} \\
& x_{2}^{\prime}(t)=-\left(1+\sin ^{2} t\right) x_{2}(t)+\frac{1}{4}\left(1+\cos ^{2} t\right) x_{1}(t)+\beta_{2} x_{2}\left(t-\tau_{2 j}(t)\right) e^{-c_{2 j}(t) x_{2}\left(t-\tau_{2 j}(t)\right)}
\end{aligned}
$$


for any $0<\beta_{2} \leq \frac{1}{2}$.

Remark 3.3. In recent years, some attention has been given to Nicholson's blowflies equations and systems with harvesting. For the $n$-dimensional case, such systems are obtained by adding linear harvesting terms with delays to (3.5), so that it becomes:

$$
\begin{aligned}
x_{i}^{\prime}(t)= & -d_{i}(t) x_{i}(t)+\sum_{j=1, j \neq i}^{n} a_{i j}(t) x_{j}(t) \\
& +\sum_{k=1}^{m} \beta_{i k}(t) x_{i}\left(t-\tau_{i k}(t)\right) e^{-c_{i k}(t) x_{i}\left(t-\tau_{i k}(t)\right)}-H_{i}(t) x_{i}\left(t-\sigma_{i}(t)\right), \quad i=1, \ldots, n,
\end{aligned}
$$

where the new coefficients $H_{i}(t)$ and delays $\sigma_{i}(t)$ are continuous, nonnegative and bounded. For the scalar case of (3.25), Liu [18] studied both the global exponential stability of the zero solution and the permanence. The almost periodic scalar case of (3.25) was studied in [30], and the $n$-dimensional case in [28], where the authors established criteria for the existence and global exponential stability of a positive almost periodic solution by using properties of almost periodic functions and Lyapunov functionals. See also [31] for a periodic system (3.25) with $n=2$. From the proof of Theorem 3.2, we deduce that Theorem 3.5. (ii), on the global asymptotic stability of the zero solution, applies to (3.5) replaced by (3.25), without any changes. However, the result on permanence in Theorem 3.5. (iii) does not carry over to (3.25). An interesting open problem is to generalize our results, and find sufficient conditions for the permanence of (3.25).

Remark 3.4. In [9], Faria studied the persistence and permanence of a class of cooperative DDEs with possible infinite delay of the form $x_{i}^{\prime}(t)=F_{i}\left(x_{t}\right)-x_{i}(t) G_{i}\left(x_{t}\right), 1 \leq i \leq n$. By using properties of cooperative systems, it was shown that, under some additional conditions, all positive solutions are bounded below and above by positive equilibria, which in particular proves the permanence. The persistence and permanence for the non-autonomous system $x_{i}^{\prime}(t)=F_{i}\left(t, x_{t}\right)-x_{i}(t) G_{i}\left(t, x_{t}\right), 1 \leq i \leq$ $n$, was also addressed in [9] by comparing it above and below with autonomous cooperative systems. Although the basic idea is similar (comparison of solutions with solutions of cooperative systems), the results and techniques in [9] do not apply to the study of systems (3.1): not only does (3.1) not have the above form, but the nonlinearities $h_{i k}(t, x)$ are in general non-monotone on the second variable. On the other hand, this remark raises another interesting open problem: how to extend the results about permanence in this paper to systems with infinite delay, since it is clear that the proof of Theorem 3.3 does not work for the infinite delay case.

\section{Sharp criteria for systems with autonomous coefficients}

The case of an autonomous system (3.1), or of (3.1) with constant coefficients but time-varying delays, is particularly important in applications. For these situations, the matrices $A, B, D, M$ in (3.4) are autonomous, and their properties play an important role in the analysis of the asymptotic behaviour of solutions. For the sake of completeness and convenience of the reader, some elements from matrix theory will be recalled here. We start with some definitions. 
Definition 4.1. Let $N=\left[n_{i j}\right]$ be a square matrix. The matrix $N$ is said to be reducible if there is a simultaneous permutation of rows and columns that brings $N$ to the form

$$
\left[\begin{array}{cc}
N_{11} & 0 \\
N_{21} & N_{22}
\end{array}\right]
$$

with $N_{11}$ and $N_{22}$ square matrices; $N$ is an irreducible matrix if it is not reducible. For $N$ with nonpositive off-diagonal entries (i.e., $n_{i j} \leq 0$ for $i \neq j$ ), $N$ is said to be a non-singular M-matrix if all its eigenvalues have positive real parts. We say that $N$ is a cooperative matrix if it has nonnegative off-diagonal entries (i.e., $n_{i j} \geq 0$ for $i \neq j$ ).

The reader should be aware that many authors use the term $M$-matrix with the above meaning of the term non-singular M-matrix. For alternative definitions and properties of M-matrices, see [11]. Namely, it is important to remark that, for a square matrix $N$ with nonpositive off-diagonal entries, $N$ is a non-singular M-matrix if and only if there exists a vector $u \gg 0$ such that $N u \gg 0$.

System (3.1) with constant coefficients becomes

$$
x_{i}^{\prime}(t)=-d_{i} x_{i}(t)+\sum_{j=1, j \neq i}^{n} a_{i j} x_{j}(t)+\sum_{k=1}^{m} \beta_{i k} h_{i k}\left(x_{i}\left(t-\tau_{i k}(t)\right)\right), i=1, \ldots, n, t \geq 0,
$$

and hypothesis (h4) translates simply as

$\left(\mathrm{h} 4^{*}\right) h_{i k}:[0, \infty) \rightarrow[0, \infty)$ are bounded, locally Lipschitzian and continuously differentiable on a vicinity of $0^{+}$, with $h_{i k}(0)=0, h_{i k}^{\prime}(0)=1$ and $h_{i k}(x)>0$ for $x>0, i \in\{1, \ldots, n\}, k \in$ $\{1, \ldots, m\}$.

For (4.1), the results in the previous section are summed up in the following theorem:

Theorem 4.1. Consider system (4.1), where $d_{i}>0, a_{i j} \geq 0, \beta_{i k} \geq 0$ with $\beta_{i}:=\sum_{k=1}^{m} \beta_{i k}>0$, $h_{i k}, \tau_{i k}:[0, \infty) \rightarrow[0, \infty)$ are continuous, with $\tau_{i k}(t)$ uniformly bounded from above by some $\tau>0$, $i, j=1, \ldots, n, k=1, \ldots, m$, and suppose that $\left(h_{4}^{*}\right)$ is satisfied. Define the $n \times n$ matrices

$$
A=\left[a_{i j}\right], B=\operatorname{diag}\left(\beta_{1}, \ldots, \beta_{n}\right), D=\operatorname{diag}\left(d_{1}, \ldots, d_{n}\right), M=B-D+A,
$$

where $a_{i i}:=0(1 \leq i \leq n)$, and assume that $D-A$ is a non-singular $M$-matrix. Then:

(i) (4.1) is dissipative;

(ii) If in addition $h_{i}^{+}(x):=\max _{1 \leq k \leq m} h_{i k}(x)<x$ for $x>0, i=1, \ldots, n$, and there exists a vector $v \gg 0$ such that $M v \leq 0$, the trivial solution of (4.1) is GAS;

(iii) If there exists a vector $v \gg 0$ such that $M v \gg 0$, (4.1) is permanent.

For an $n \times n$ matrix $N$, the spectral bound or stability modulus $s(N)$ is defined by

$$
s(N)=\{\operatorname{Re} \lambda: \lambda \in \sigma(N)\},
$$

where $\sigma(N)$ denotes the spectrum of $N$. For a cooperative and irreducible matrix $N$, it is well-known that the spectral bound $s(N)$ is a (simple) eigenvalue, with a strictly positive associated eigenvector, see Appendix A.5 of [26]; moreover, $s(N)>0$ if and only if there exists a strictly positive vector $v \in \mathbb{R}^{n}$ with $N v \gg 0$ [10]. Thus, a threshold criterion of permanence versus extinction is obtained from Theorem 4.1 when $A$ is an irreducible matrix. 
Corollary 4.1. Assume all the general hypotheses of Theorem 4.1 (including $\left(h_{4}^{*}\right)$ and that $D-A$ is a non-singular M-matrix) are satisfied. Further assume that $A$ is irreducible and $h_{i}^{+}(x)<x$ for $x>0, i=1, \ldots, n$. Then: (i) if $s(M) \leq 0$, the trivial solution of (4.1) is $G A S$; (ii) if $s(M)>0$, (4.1) is permanent.

This threshold criterion is not valid, in general, when $A$ (and therefore $M$ as well) is reducible. Our next task is to replace the assumptions in Theorem 4.1 by sharp conditions for extinction versus permanence when $M$ is reducible. We emphasize that usually the case of a reducible community matrix $M$ is not treated in the literature.

By an adequate simultaneous permutation of rows and columns, which amounts to a permutation of the variables in the original system (4.1), we may suppose that the $n \times n$-matrix $A$ has been transformed into the triangular form

$$
A=\left[\begin{array}{cccc}
A_{11} & 0 & \ldots & 0 \\
A_{21} & A_{22} & \ldots & 0 \\
\vdots & \vdots & \ddots & \vdots \\
A_{k 1} & A_{k 2} & \ldots & A_{k k}
\end{array}\right]
$$

where the diagonal blocks $A_{11}, \ldots, A_{k k}$ are square matrices of size $n_{1}, \ldots, n_{k}$ respectively, $n_{1}+\cdots+$ $n_{k}=n$, and are irreducible. Clearly, $k=1$ if $A$ is irreducible. Observe that a square $n \times n$-matrix $A=\left[a_{i j}\right]$ is irreducible if and only if for any nonempty proper subset $I \subset\{1, \ldots, n\}$ there are $i \in I$ and $j \in\{1, \ldots, n\} \backslash I$ such that $a_{i j} \neq 0$.

The next result extends Corollary 4.1 and gives necessary and sufficient conditions for both the uniform persistence and the global asymptotic stability of the zero solution of (4.1), in the case of a reducible matrix $A$. The result for uniform persistence was inspired by [21].

Theorem 4.2. Consider system (4.1) where $d_{i}>0, a_{i j} \geq 0, \beta_{i k} \geq 0$ with $\beta_{i}:=\sum_{k=1}^{m} \beta_{i k}>0$, $\tau_{i k}:[0, \infty) \rightarrow[0, \infty)$ are continuous and bounded, the functions $h_{i k}$ satisfy $\left(h_{4}^{*}\right)$ with $h_{i}^{+}(x):=$ $\max _{1 \leq k \leq m} h_{i k}(x)<x$ for any $x>0$, for $i, j=1, \ldots, n, k=1, \ldots, m$. Let $A, B, D, M$ be the matrices defined in (4.2). Assume that $D-A$ is a non-singular $M$-matrix. Without loss of generality, further assume that $A$ has the block lower triangular structure as in (4.3), with irreducible diagonal blocks $A_{11}, \ldots, A_{k k}$, and denote by $M_{j j}$ the associated blocks in the matrix $M$, that is, $M_{j j}=B_{j}-D_{j}+$ $A_{j j}$, with $B_{j}=\operatorname{diag}\left(\beta_{i}\right)_{i \in I_{j}}$ and $D_{j}=\operatorname{diag}\left(d_{i}\right)_{i \in I_{j}}$, where $I_{j}$ is the set formed by the $n_{j}$ indexes corresponding to the rows of the block $A_{j j}$, for each $j=1, \ldots, k$; and $M_{i j}=A_{i j}$ for $1 \leq j<i \leq k$. Then:

(i) System (4.1) is uniformly persistent if and only if $s\left(M_{j j}\right)>0$ for every index $j \in\{1, \ldots, k\}$ such that, except for the diagonal block $M_{j j}$, all the other blocks on the row are null.

(ii) The null solution of system (4.1) is GAS if and only if $s(M) \leq 0$.

Proof. In the case of $A$ irreducible, the results are given in Corollary 4.1. From now on, $A$ is assumed to be a reducible matrix with the triangular form (4.3) with $k>1$. We make a few remarks beforehand.

First, observe that the property of $D-A$ being a non-singular M-matrix is preserved under a simultaneous permutation of rows and columns (so that $D-A$ becomes $P(D-A) P^{T}$ for some orthogonal matrix $P$ ), therefore system (4.1) is dissipative, and thus the uniform persistence in (i) 
can actually be replaced by the permanence. Secondly, for each $j=1, \ldots, k$, we consider the lower dimensional system associated with the irreducible block $M_{j j}$, formed by the $n_{j}$ equations

$$
x_{i}^{\prime}(t)=-d_{i} x_{i}(t)+\sum_{p \in I_{j}, p \neq i} a_{i p} x_{p}(t)+\sum_{q=1}^{m} \beta_{i q} h_{i q}\left(x_{i}\left(t-\tau_{i q}(t)\right)\right), i \in I_{j}, t \geq 0,
$$

and observe that it satisfies all the hypotheses in Corollary 4.1, as $D_{j}-A_{j j}$ is a non-singular Mmatrix as well. Finally, for any vector $v$ in $\mathbb{R}^{n}$ or any map $v$ taking values in $\mathbb{R}^{n}$, we introduce the notation $v^{j}=\left(v_{i}\right)_{i \in I_{j}}$ for each $j=1, \ldots, k$, so that $v=\left(v^{1}, \ldots, v^{k}\right)$.

(i) To simplify the writing, we may assume without loss of generality that the diagonal blocks in (4.3) with all null blocks to their left (if any) are placed in the first rows. In other words, we assume that $\left\{A_{11}, \ldots, A_{l l}\right\}$ are exactly the diagonal blocks with all other blocks on their row null, for some $1 \leq l \leq k$. In this way, for $1 \leq j \leq l$, system (4.4) is just a lower dimensional decoupled subsystem of system (4.1).

Now, suppose that system (4.1) is uniformly persistent. Then, for each $j=1, \ldots, l$, system (4.4) naturally inherits the property of uniform persistence from the total system, and Corollary 4.1]implies that $s\left(M_{j j}\right)>0$ for any $j=1, \ldots, l$, so this is a necessary condition.

Conversely, assume that $s\left(M_{j j}\right)>0$ for any $j=1, \ldots, l$. Applying once more Corollary 4.1, we deduce that systems (4.4) are uniformly persistent for any $j=1, \ldots, l$. Therefore, there exists $m_{0}>0$ such that for any $\phi \in C_{0}, \liminf _{t \rightarrow \infty} x_{i}(t, 0, \phi) \geq m_{0}$ for all $i \in I_{1} \cup \ldots \cup I_{l}$. At this point, if $l=k$ the proof is complete, whereas if $l<k$ we have to deal with the remaining components of the solution.

We now consider the case $l<k$ and look at the components $x_{i}(t, 0, \phi)$ for $i \in I_{l+1}$. The method here is twofold: first, since there is at least a non-null block to the left of $M_{l+1, l+1}$, we will show that one component $x_{i_{1}}(t, 0, \phi)\left(i_{1} \in I_{l+1}\right)$ of the solution eventually stays bounded away from 0 . Secondly, once we have raised one component in $I_{l+1}$, we recursively raise the rest of them, one by one, by applying the irreducible character of $M_{l+1, l+1}$.

More precisely, as there is at least one non-null block to the left of $M_{l+1, l+1}$, there are indexes $i_{1} \in I_{l+1}$ and $j_{1} \in I_{j}$ for some $1 \leq j \leq l$ such that $a_{i_{1} j_{1}}>0$. Now, for an initial condition $\phi \in C_{0}$, there exists a $t_{0}=t_{0}(\phi)$ such that $x_{i}(t, 0, \phi) \geq m_{0}$ for all $t \geq t_{0}$ and for all $i \in I_{1} \cup \ldots \cup I_{l}$. Therefore, for $t \geq t_{0}, x_{i_{1}}^{\prime}(t, 0, \phi) \geq-d_{i_{1}} x_{i_{1}}(t, 0, \phi)+a_{i_{1} j_{1}} m_{0}$. Now, we consider the scalar cooperative ODE

$$
y^{\prime}(t)=-d_{i_{1}} y(t)+a_{i_{1} j_{1}} m_{0}, t \geq 0,
$$

whose solution, for the previous time $t_{0} \geq 0$, is written as

$$
y\left(t, t_{0}, y\left(t_{0}\right)\right)=y\left(t_{0}\right) e^{-d_{i_{1}}\left(t-t_{0}\right)}+\frac{a_{i_{1} j_{1}} m_{0}}{d_{i_{1}}}\left(1-e^{-d_{i_{1}}\left(t-t_{0}\right)}\right),
$$

so that there exist $m_{1}>0$ and $t_{1} \geq t_{0}$ such that $y\left(t, t_{0}, y\left(t_{0}\right)\right) \geq m_{1}$ for any $t \geq t_{1}$, provided that $y\left(t_{0}\right) \geq 0$. The application of a standard argument of comparison of solutions permits to conclude that $x_{i_{1}}(t, 0, \phi) \geq m_{1}$ for any $t \geq t_{1}$.

If $I_{l+1}=\left\{i_{1}\right\}$, we are done with this block. If not, as $A_{l+1, l+1}$ is irreducible, there exists an index $i_{2} \in I_{l+1} \backslash\left\{i_{1}\right\}$ such that $a_{i_{2} i_{1}}>0$. As before, we consider the scalar ODE

$$
y^{\prime}(t)=-d_{i_{2}} y(t)+a_{i_{2} i_{1}} m_{1}, t \geq 0,
$$


for which we find a constant $m_{2}>0$ and a time $t_{2} \geq t_{1}$ such that if $t \geq t_{2}, y\left(t, 0, y\left(t_{1}\right)\right) \geq m_{2}$ for any $t \geq t_{2}$, independently of the value $y\left(t_{1}\right) \geq 0$. In a similar way, we conclude that the $i_{2}$ th component of the solution of (4.1) satisfies $x_{i_{2}}^{\prime}(t, 0, \phi) \geq-d_{i_{2}} x_{i_{2}}(t, 0, \phi)+a_{i_{2} i_{1}} m_{1}$ for any $t \geq t_{1}$, and once more, by comparing solutions, we have $x_{i_{2}}(t, 0, \phi) \geq m_{2}$ for any $t \geq t_{2}$.

At this point, if $I_{l+1}=\left\{i_{1}, i_{2}\right\}$ we are finished with this block; if not, as $A_{l+1, l+1}$ is irreducible, considering $\left\{i_{1}, i_{2}\right\}$ and its complement $I_{l+1} \backslash\left\{i_{1}, i_{2}\right\}$, we may affirm that there exist indexes $i_{3} \in$ $I_{l+1} \backslash\left\{i_{1}, i_{2}\right\}$ and $j \in\left\{i_{1}, i_{2}\right\}$ such that $a_{i_{3} j}>0$; now, the argument to lift the component $x_{i_{3}}(t, 0, \phi)$ is just the same as the one for $x_{i_{2}}(t, 0, \phi)$.

Iterating this procedure inside the irreducible block $A_{l+1, l+1}$, we conclude that there is a constant $m_{0}^{\prime}=\min \left\{m_{0}, m_{1}, \ldots, m_{n_{l+1}}\right\}>0$ such that for any $\phi \in C_{0}$, there exists a $t_{0}^{\prime}=t_{0}^{\prime}(\phi)$ such that $x_{i}(t, 0, \phi) \geq m_{0}^{\prime}$ for all $t \geq t_{0}^{\prime}$ and for all $i \in I_{1} \cup \ldots \cup I_{l} \cup I_{l+1}$.

To finish, note that the procedure for the remaining components of the solution, if any, is identical to the one just developed for the set of indexes $I_{l+1}$.

(ii) Note that $s(M)=\max \left\{s\left(M_{11}\right), \ldots, s\left(M_{k k}\right)\right\}$, so that $s(M) \leq 0$ if and only if $s\left(M_{j j}\right) \leq 0$ for $j=1, \ldots, k$. Because of the triangular structure of $A$ in (4.3), and with the previous notation for $\phi=\left(\phi^{1}, \ldots, \phi^{k}\right)$, it is apparent that, for $j=2, \ldots, k$, the "faces"

$$
F_{j}=\left\{\phi=\left(\phi^{1}, \ldots, \phi^{k}\right) \in C^{+} \mid \phi^{1}=\ldots=\phi^{j-1}=0\right\}
$$

of the nonnegative cone $C^{+}$are positively invariant. In this way, for an initial condition $\phi \in F_{j}(2 \leq$ $j \leq k)$, the solution remains in $F_{j}$, thus the component $x^{j}(t, 0, \phi)$ is a solution of the system (4.4).

We first assume that the null solution of (4.1) is GAS in the nonnegative cone $C^{+}$. For any $j=1, \ldots, k$ fixed, we now show that any solution $y\left(t, 0, \phi^{j}\right)$ of system (4.4) with initial condition $\phi^{j} \in C^{+}\left([-\tau, 0] ; \mathbb{R}^{n_{j}}\right)$ has $\lim _{t \rightarrow \infty} y\left(t, 0, \phi^{j}\right)=0$. This is clear for $j=1$, as system (4.4) is a decoupled subsystem of system (4.1). For $j>1$, just consider $\widetilde{\phi} \in C^{+}\left([-\tau, 0] ; \mathbb{R}^{n}\right)$ with $\widetilde{\phi}^{j}=\phi^{j}$ and $\widetilde{\phi}^{i}=0$ for any $i<j$, so that $\widetilde{\phi} \in F_{j}$. Then, $x^{j}(t, 0, \widetilde{\phi})$ is a solution of system (4.4), thus $y\left(t, 0, \phi^{j}\right)=x^{j}(t, 0, \widetilde{\phi}) \rightarrow 0$ as $t \rightarrow \infty$, as we wanted. With this behaviour for each $j$, the persistent case in Corollary 4.1 is precluded, and then it must be $s\left(M_{j j}\right) \leq 0$.

Conversely, assume that $s\left(M_{j j}\right) \leq 0$, so that there exist vectors $v^{j} \in \mathbb{R}^{n_{j}}, v_{j} \gg 0$ such that $M_{j j} v^{j} \leq 0$ for $j=1, \ldots, k$. Without loss of generality, we suppose that $k=2$, so that $M$ has the form

$$
M=B-D+\left[\begin{array}{cc}
A_{11} & 0 \\
A_{21} & A_{22}
\end{array}\right],
$$

where $A_{i i}$ are $n_{i} \times n_{i}$ irreducible blocks $(i=1,2)$. The general case of $k$ blocks follows by iterating the procedure below.

With $M$ given by (4.5), (4.1) is equivalent to

$$
\begin{aligned}
& x_{i}^{\prime}(t)=-d_{i} x_{i}(t)+\sum_{p \in I_{1}, p \neq i} a_{i p} x_{p}(t)+\sum_{k=1}^{m} \beta_{i k} h_{i k}\left(x_{i}\left(t-\tau_{i k}(t)\right)\right), i \in I_{1}, t \geq 0 \\
& x_{i}^{\prime}(t)=-d_{i} x_{i}(t)+\sum_{p \in I_{2}, p \neq i} a_{i p} x_{p}(t)+\sum_{k=1}^{m} \beta_{i k} h_{i k}\left(x_{i}\left(t-\tau_{i k}(t)\right)\right)+\sum_{p \in I_{1}} a_{i p} x_{p}(t), i \in I_{2}, t \geq 0 .
\end{aligned}
$$


We first claim that the trivial solution of (4.1) is globally attractive. Let $\phi \in C^{+}$, and write $x(t, 0, \phi)=\left(x^{1}\left(t, 0, \phi^{1}\right), x^{2}(t, 0, \phi)\right)$. (Recall that $x^{1}(t, 0, \phi)$ is just the solution of system (4.4) for $j=1$ with initial condition $\phi^{1}$.) Corollary 4.1 implies that $\lim _{t \rightarrow \infty} x^{1}\left(t, 0, \phi^{1}\right)=0$. In particular, in (4.6) we have $q_{i}(t):=\sum_{p \in I_{1}} a_{i p} x_{p}\left(t, 0, \phi^{1}\right) \rightarrow 0$ as $t \rightarrow \infty$ for any $i \in I_{2}$. At this point, the proof of $\lim _{t \rightarrow \infty} x^{2}(t, 0, \phi)=0$ is obtained by simply repeating the argument used in the proof of Theorem 3.2 applied to the second system in (4.6). Details are omitted.

It remains to prove the stability of the null solution of (4.6). For a given $\phi=\left(\phi^{1}, \phi^{2}\right) \in C^{+}$, as before we denote the solution of (4.1) by $x(t, 0, \phi)=\left(x^{1}\left(t, 0, \phi^{1}\right), x^{2}(t, 0, \phi)\right)$.

From the assumptions on $h_{i k}$, for $i \in I_{2}$, we construct maps $\widetilde{h}_{i}:[0, \infty) \rightarrow[0, \infty)$ satisfying the following conditions: $\widetilde{h}_{i}$ are continuous, bounded, nondecreasing, equal to $h_{i}^{+}$on a right neighbourhood of 0 , and such that $h_{i}^{+}(x) \leq \widetilde{h}_{i}(x)<x$ for all $x>0$. Now, we consider an $n$-dimensional system, whose first $n_{1}$ equations are given by (4.4) with $j=1$ (as in (4.6)) and the last equations given by the $n_{2}$-dimensional system

$$
y_{i}^{\prime}(t)=-d_{i} y_{i}(t)+\sum_{p \in I_{2}, p \neq i} a_{i p} y_{p}(t)+\sum_{k=1}^{m} \beta_{i k} \widetilde{h}_{i}\left(y_{i}\left(t-\tau_{i k}(t)\right)\right)+\sum_{p \in I_{1}} a_{i p} x_{p}(t), i \in I_{2}, t \geq 0,
$$

written for short as $y_{i}^{\prime}(t)=\widetilde{f}_{i}\left(t, y_{t}\right)$. Since (4.7) is cooperative, a comparison of solutions leads to $x^{2}(t, 0, \phi) \leq y(t, 0, \phi, \widetilde{f})$ for $t \geq 0$.

Fix any $\varepsilon>0$. Let $\widetilde{\varepsilon}>0$ be sufficiently small so that $\beta_{i}\left(\varepsilon v_{i}^{2}-\widetilde{h}_{i}\left(\varepsilon v_{i}^{2}\right)\right) \geq \widetilde{\varepsilon} v_{i}^{2}, i \in I_{2}$. Of course, Corollary 4.1 (or Theorem 3.2) yields the stability of the null solution for the first system in (4.6), thus there is $\delta_{1}=\delta_{1}(\varepsilon)>0$ such that $0 \leq x^{1}\left(t, 0, \phi^{1}\right) \leq \varepsilon v^{1}$ for $t \geq 0$ whenever $0 \leq \phi^{1} \leq \delta_{1} v^{1}$. Moreover, we find $\widetilde{\delta}_{1}=\widetilde{\delta}_{1}(\widetilde{\varepsilon})=\widetilde{\delta}_{1}(\varepsilon)>0$ such that if $0 \leq \phi^{1} \leq \widetilde{\delta}_{1} v^{1}$, then $0 \leq A_{21} x^{1}\left(t, 0, \phi^{1}\right) \leq \widetilde{\varepsilon} v^{2}$ for $t \geq 0$.

Take $\delta=\min \left(\delta_{1}, \widetilde{\delta}_{1}\right)$, and consider an initial condition $\phi \in C^{+}$with $0 \leq \phi^{1} \leq \delta v^{1}$ and $0 \leq$ $\phi^{2} \leq \varepsilon v^{2}$. We first solve the decoupled $n_{1}$-dimensional system, and replace in (4.7) the terms $\sum_{p \in I_{1}} a_{i p} x_{p}(t)$ by $\sum_{p \in I_{1}} a_{i p} x_{p}\left(t, 0, \phi^{1}\right)$. The crucial point is to check that $\varepsilon v^{2}$ is an "upper" solution for this new cooperative system, or in other words, that $\widetilde{f}\left(t, \varepsilon v^{2}\right) \leq 0$ for any $t \geq 0$; this allows concluding that the set $\left[0, \varepsilon v^{2}\right] \subset C\left([-\tau, 0] ; \mathbb{R}^{n_{2}}\right)$ is positively invariant for (4.7) (see Lemma 2.3). For each $i \in I_{2}$, we have

$$
\begin{aligned}
\widetilde{f}_{i}\left(t, \varepsilon v^{2}\right) & =-d_{i} \varepsilon v_{i}^{2}+\sum_{j \in I_{2}, j \neq i} a_{i j} \varepsilon v_{j}^{2}+\beta_{i} \widetilde{h}_{i}\left(\varepsilon v_{i}^{2}\right)+\sum_{j \in I_{1}} a_{i j} x_{j}^{1}\left(t, 0, \phi^{1}\right) \\
& =-d_{i} \varepsilon v_{i}^{2}+\sum_{j \in I_{2}, j \neq i} a_{i j} \varepsilon v_{j}^{2}+\beta_{i} \varepsilon v_{i}^{2}+\beta_{i}\left(\widetilde{h}_{i}\left(\varepsilon v_{i}^{2}\right)-\varepsilon v_{i}^{2}\right)+\sum_{j \in I_{1}} a_{i j} x_{j}^{1}\left(t, 0, \phi^{1}\right),
\end{aligned}
$$

hence,

$$
\widetilde{f}\left(t, \varepsilon v^{2}\right) \leq \varepsilon M_{22} v^{2}-\widetilde{\varepsilon} v^{2}+\widetilde{\varepsilon} v^{2} \leq 0 .
$$

As a consequence, and summarizing, we deduce that, whenever $0 \leq \phi^{1} \leq \delta v^{1}$ and $0 \leq \phi^{2} \leq \varepsilon v^{2}$ then $0 \leq x^{1}\left(t, 0, \phi^{1}\right) \leq \varepsilon v^{1}$ and $0 \leq x^{2}\left(t, 0, \phi^{1}, \phi^{2}\right) \leq y\left(t, 0, \phi^{1}, \phi^{2}\right) \leq \varepsilon v^{2}$ for $t \geq 0$. This ends the proof.

Theorem 4.2 also provides conditions for partial extinction and partial persistence. As an illustration, we summarise the results for a Nicholson system. 
Example 4.1. Consider the Nicholson system with autonomous coefficients and time-dependent delays given by

$$
x_{i}^{\prime}(t)=-d_{i} x_{i}(t)+\sum_{j=1, j \neq i}^{n} a_{i j} x_{j}(t)+\sum_{k=1}^{m} \beta_{i k} x_{i}\left(t-\tau_{i k}(t)\right) e^{-c_{i k} x_{i}\left(t-\tau_{i k}(t)\right)}, i=1, \ldots, n, t \geq 0,
$$

where $d_{i}>0, c_{i k}>0, a_{i j} \geq 0, \beta_{i k} \geq 0$ with $\beta_{i}:=\sum_{k=1}^{m} \beta_{i k}>0, \tau_{i k}:[0, \infty) \rightarrow[0, \tau](\tau>0)$ are continuous, for all $i, j, k$, and $D-A$ is a non-singular M-matrix. By applying Theorem 4.2 to this model, we obtain:

(i) if $s(M) \leq 0,0$ is GAS;

(ii) if $M$ is written in the triangular form (for some $k \in\{1, \ldots, n\}$ and some $l \in\{1, \ldots, k\}$ )

$$
M=\left[\begin{array}{cccccc}
M_{11} & \ldots & 0 & 0 & \ldots & 0 \\
\vdots & \ddots & \vdots & \vdots & & \vdots \\
0 & \ldots & M_{l l} & 0 & \ldots & 0 \\
M_{l+1,1} & \ldots & M_{l+1, l} & M_{l+1, l+1} & \ldots & 0 \\
\vdots & & \vdots & \vdots & \ddots & \vdots \\
M_{k 1} & \ldots & M_{k, l} & M_{k, l+1} & \ldots & M_{k k}
\end{array}\right]
$$

with $M_{j j}(1 \leq j \leq k)$ irreducible blocks and $M_{j p} \neq 0$ for some $p<j$ and $j=l+1, \ldots k$, then (4.8) is permanent if and only if $s\left(M_{j j}\right)>0$ for $j=1, \ldots, l$;

(iii) moreover, for $M$ written in the triangular form (4.9), if $l>1$ and there exist $p, j \in\{1, \ldots, l\}$ such that $s\left(M_{p p}\right) \leq 0$ and $s\left(M_{j j}\right)>0$, then the $n_{p}$ populations $x_{i}(t)$ with $i \in I_{p}$ become extinct, whereas the $n_{j}$ populations $x_{i}(t)$ with $i \in I_{j}$ uniformly persist.

Remark 4.1. In this way, we have recovered and extended all the results regarding extinction and uniform persistence established in [7, 10] for the particular case of (4.8) with constant delays $\tau_{i k}$ and $c_{i k}=1$ for all $i, k$. For such autonomous systems, the sharp criterion for extinction of all populations, $s(M) \leq 0$, was proven in [10] by using the unimodal shape of the specific Ricker nonlinearity $h(x)=x e^{-x}$. However, as shown in the proof of Theorem 4.2, the techniques presented in Section 3, based on comparison of solutions with solutions for auxiliary cooperative systems, allow us to carry out the arguments for the more general model (4.1). Also, the permanence of Nicholson autonomous systems in [10] was proven under the stronger requirement of $M v \gg 0$ for some $v \gg 0$ (and $D-A$ a non-singular M-matrix).

\section{Acknowledgements}

This work was partially supported by Fundação para a Ciência e a Tecnologia under project UID/MAT/04561/2013 (T. Faria) and by Ministerio de Economía y Competitividad under project MTM201566330, and the European Commission under project H2020-MSCA-ITN-2014 (R. Obaya and A. M. Sanz).

The authors are very grateful to the referee, whose careful reading and valuable comments led to significant improvements of the manuscript. 


\section{References}

[1] Bélair, J., Mackey, M., Mahaffy, J.: Age-structured and two-delays models for erythropoiesis, Math. Biosci. 241, 109-119 (2006).

[2] Berezansky, L., Braverman, E.: Boundedness and persistence of delay differential equations with mixed nonlinearity, Appl. Math. Comput. 279, 154-169 (2016).

[3] Berezansky, L., Braverman, E., Idels, L.: Nicholson's blowflies differential equations revisited: main results and open problems, Appl. Math. Model. 34, 1405-1417 (2010).

[4] Berezansky, L., Idels, L., Troib, L.: Global dynamics of Nicholson-type delay systems with applications, Nonlinear Anal. RWA 12, 436-445 (2011).

[5] Coppel, W.A.: Dichotomies in Stability Theory, Lecture Notes in Mathematics, Vol. 629, Springer-Verlag, Berlin-New York (1978).

[6] Cushing, J.M.: An Introduction to Structured Population Dynamics, Conference Series in Applied Mathematics Vol 71, SIAM, Philadelphia (1998).

[7] Faria, T.: Global asymptotic behaviour for a Nicholson model with patch structure and multiple delays, Nonlinear Anal. 74, 7033-7046 (2011).

[8] Faria, T.: Global dynamics for Lotka-Volterra systems with infinite delay and patch structure, Appl. Math. Comput. 245, 575-590 (2014) .

[9] Faria, T.: Persistence and permanence for a class of functional differential equations with infinite delay, J. Dyn. Diff. Equ. 28, 1163-1186 (2016).

[10] Faria, T., Röst, G.: Persistence, permanence and global stability of an $n$-dimensional Nicholson system, J. Dyn. Diff. Equ. 26, 723-744 (2014).

[11] Fiedler, M.: Special Matrices and Their Applications in Numerical Mathematics, Martinus Nijhoff Publ. (Kluwer), Dordrechit (1986).

[12] Fink, A.M.: Almost Periodic Differential Equations, Lecture Notes in Math. 377, Springer- Verlag, Berlin (1974).

[13] Gurney, W.S.C., Blythe, S.P., Nisbet, R.M.: Nicholson's blowflies revisited, Nature 287, 17-21 (1980).

[14] Hale, J. K.: Ordinary Differential Equations, John Wiley \& Sons, Inc., New York (1969).

[15] Liu, B.: Global stability of a class of delay differential equations, J. Comput. Appl. Math. 233, 217-223 (2009).

[16] Liu, B.: Global stability of a class of Nicholson's blowflies model with patch structure and multiple time-varying delays, Nonlinear Anal. RWA 11, 2557-2562 (2010).

[17] Liu, B.: The existence and uniqueness of positive periodic solutions of Nicholson-type delay systems, Nonlinear Anal. RWA 12, 3145-3451 (2011).

[18] Liu, B.: Global dynamic behaviors for a delayed Nicholson's blowflies model with a linear harvesting term, Electron. J. Qual. Theory Differ. Equ. 2013 No. 45, 1-13 (2013). 
[19] Mackey, M.C., Glass, L.: Oscillations and chaos in physiological control systems, Science 197, no.4300, 287-289 (1997).

[20] Metz, J.A.J., Diekmann, O.: The Dynamics of Physiologically Structured Populations, Lecture Notes in Biomath. 68, Springer-Verlag (1986).

[21] Novo, S., Obaya, R., Sanz, A.M.: Uniform persistence and upper Lyapunov exponents for monotone skew-product semiflows, Nonlinearity 26, 1-32 (2013).

[22] Obaya, R., Sanz, A.M.: Uniform and strict persistence in monotone skew-product semiflows with applications to non-autonomous Nicholson systems, J. Differential Equations 261, 4135- 4163 (2016).

[23] Sacker, R.J., Sell, G.R.: A spectral theory for linear differential systems, J. Differential Equations 27, 320-358 (1978).

[24] Smith, H.L.: Monotone Dynamical Systems. An Introduction to the Theory of Competitive and Cooperative Systems, Mathematical Surveys and Monographs, Amer. Math. Soc., Providence, RI (1995).

[25] Smith, H.L.: An Introduction to Delay Differential Equations with Applications to Life Sciences, Texts in Applied Mathematics Vol. 57, Springer, Berlin (2011).

[26] Smith, H.L., Thieme, H.R.: Dynamical Systems and Population Persistence, Amer. Math. Soc., Providence, RI (2011).

[27] Takeuchi, Y., Cui, J., Miyazaki, R., Saito, Y.: Permanence of delayed population model with dispersal loss, Math. Biosci. 201, 143-156 (2006).

[28] Wang, L.: Almost periodic solution for Nicholson's blowflies model with patch structure and linear harvesting terms, Appl. Math. Model. 37, 2153-2165 (2013).

[29] Wang, W., Wang, L., Chen, W.: Existence and exponential stability of positive almost periodic solution for Nicholson-type delay systems, Nonlinear Analysis RWA 12, 1938-1949 (2011).

[30] Zhang, A.: New results on almost periodic solutions for a Nicholson's blowflies model with a linear harvesting term, Electron. J. Qual. Theory Differ. Equ. 2014, No. 37, 1-14 (2014).

[31] Zhou, Q.: The positive periodic solution for Nicholson-type delay system with linear harvesting terms, Appl. Math. Model. 37 5581-5590 (2013). 